\title{
Single-top final states as a probe of top-flavoured dark matter models at the LHC
}

\author{
Monika Blanke, ${ }^{a, b}$ Priscilla Pani, ${ }^{c, d}$ Giacomo Polesello ${ }^{e}$ and Giulia Rovelli ${ }^{e, f}$ \\ ${ }^{a}$ Institute for Astroparticle Physics, Karlsruhe Institute of Technology, \\ Hermann-von-Helmholtz-Platz 1, D-76344 Eggenstein-Leopoldshafen, Germany \\ ${ }^{b}$ Institute for Theoretical Particle Physics, Karlsruhe Institute of Technology, \\ Engesserstraße 7, 76131 Karlsruhe, Germany \\ ${ }^{c}$ Deutsches Elektronen-Synchrotron DESY, \\ Notkestraße 85, 22607 Hamburg, Germany \\ ${ }^{d}$ Deutsches Elektronen-Synchrotron DESY, \\ Platanenallee 6, 15738 Zeuthen, Germany \\ INFN, Sezione di Pavia, \\ Via Bassi 6, 27100 Pavia, Italy \\ ${ }^{f}$ Università degli Studi di Pavia, Dipartimento di Fisica, \\ Via Bassi 6, 27100 Pavia, Italy \\ E-mail: monika.blanke@kit.edu, priscilla.pani@cern.ch, \\ giacomo.polesello@cern.ch, giulia.rovelli@cern.ch
}

ABSTRACT: Models incorporating flavoured dark matter provide an elegant solution to the dark matter problem, evading the tight LHC and direct direction constraints on simple WIMP models. In Dark Minimal Flavour Violation, a simple framework of flavoured dark matter with new sources of flavour violation, the constraints from thermal freeze-out, direct detection experiments, and flavour physics create well-defined benchmark scenarios for these models. We study the LHC phenomenology of four such scenarios, focusing on final states where a single top quark is produced accompanied by no jets, one jet from the fragmentation of light quarks or a $b$-tagged jet. For each of these signatures we develop a realistic LHC analysis, and we show that the proposed analyses would increase the parameter space coverage for the four benchmarks, compared to existing flavour-conserving LHC analyses. Finally we show the projected discovery potential of the considered signatures for the full LHC statistics at $14 \mathrm{TeV}$, and for the High Luminosity LHC.

KEywords: Beyond Standard Model, Dark matter, Flavor physics, Hadron-Hadron scattering (experiments), Top physics

ArXiv EPrint: 2010.10530 


\section{Contents}

1 Introduction 1

2 DMFV models and definition of the benchmark scenarios 2

2.1 The DMFV framework 3

2.2 Right-handed model 3

2.3 Left-handed model 4

2.4 Phenomenology and benchmark scenarios 4

3 LHC phenomenology $\quad 5$

3.1 Pair production of mediators $\quad 6$

$\begin{array}{ll}3.2 & \text { Single top final states } \\ & 8\end{array}$

4 Recast of LHC limits for mediator pair production 11

5 Detailed analyses of single-top signatures $\quad \mathbf{1 4}$

$\begin{array}{ll}5.1 \text { Monte Carlo simulation } & 15\end{array}$

$\begin{array}{ll}5.2 \text { Statistical procedures } & 16\end{array}$

$\begin{array}{ll}5.3 \text { Analysis strategy } & 16\end{array}$

$\begin{array}{llr}6 & \text { Results } & 19\end{array}$

$\begin{array}{lll}7 & \text { Conclusion and outlook } & 22\end{array}$

\section{Introduction}

Weakly interacting massive particles (WIMPs) are among the theoretically best-motivated candidates to explain the observed dark matter (DM) density in the universe $[1,2]$. However, the absence of signal in both direct detection experiments and at the Large Hadron Collider (LHC) has put simple WIMP models under severe pressure, challenging the presence of a sufficiently large DM annihilation cross-section.

A possible way out of this dilemma is offered by the introduction of a non-trivial flavour structure in the dark sector [3-11]. In this scenario, dark matter transforms as a multiplet (usually triplet) under a flavour symmetry and couples non-universally to the different flavours of the Standard Model (SM). If the lightest dark flavour couples predominantly to the third quark generation, its interactions with the SM nuclei are reduced, thereby reconciling the thermal freeze-out condition with the experimental limits. Such models are usually referred to as top- or bottom-flavoured dark matter.

Most interesting from the phenomenological point of view are models which go beyond the assumption of Minimal Flavour Violation (MFV). In a series of papers [12-14] the 
framework of Dark Minimal Flavour Violation (DMFV) has been put forward. In this class of models, the interaction between the dark matter flavour triplet and the SM quarks, ${ }^{1}$ mediated by a coloured scalar $\phi$, constitutes the only new source of flavour violation, thus efficiently reducing the number of free parameters while at the same time conserving the rich phenomenology of a non-MFV model. The interplay of constraints from flavour physics, direct detection experiments and the thermal freeze-out condition then allows to place limits on the parameter space of the model, thereby creating benchmark scenarios to be targeted by future LHC searches.

At the LHC, DMFV models provide final state signatures involving the production of scalar mediators $(\phi)$, each of which further decays into a quark and a fermionic dark matter particle $(\chi)$. The scalar mediators can be produced in pairs, leading to signatures with two quarks and $E_{\mathrm{T}}^{\text {miss }}$, or singly produced accompanied by a dark matter particle, leading to final states with one quark and $E_{\mathrm{T}}^{\text {miss }}$.

As $\phi$ has the same quantum numbers as a supersymmetric squark, the final states for pair production, when both mediators decay to experimentally indistinguishable quark flavours are identical to flavour-conserving SUSY squark production, and limits on the parameter space of the DMFV models can be obtained by a simple recasting of the existing squark searches at the LHC. This exercise was performed on the LHC Run 1 results in [1214], yielding stringent limits on the parameter space of the model.

A specific feature of DMFV models is the flavour-violating signatures with a single quark or two quarks with different flavours. In the case of top-flavoured DM, this leads to LHC signatures featuring a single top quark accompanied by two dark matter particles, and either zero additional jets or an additional light $(u, d, s, c)$ or $b$-jet produced in the resonant decay of the mediator, on which we concentrate in this paper.

The detailed analyses of top-flavoured dark matter coupling to left- [13] or right-handed quarks [14] have identified the phenomenological sweet-spots in the parameter space of these models for which the constraints from flavour and dark matter experiments are satisfied. Based on these findings, we define for this work four benchmark classes of models for which we develop a search strategy at the LHC based on final states with a single top.

After defining the benchmarks, we study in detail their LHC phenomenology, addressing both the signatures from mediator pair production and the final states featuring a single top quark. Based on this work, we define the LHC constraints on the benchmarks by recasting recent results from the LHC experiments based on $\sim 140 \mathrm{fb}^{-1}$ of data collected at $\sqrt{s}=13 \mathrm{TeV}$. For each of the single-top signatures, we then develop a realistic analysis strategy, leading to a comparison of their potential in constraining the parameter space of the four benchmarks with the existing LHC bounds. We further predict the reach of the proposed signatures for the projected full statistics of the $\mathrm{LHC}$ at $14 \mathrm{TeV}, 300 \mathrm{fb}^{-1}$, and for the High Luminosity LHC project, $3 \mathrm{ab}^{-1}$.

\section{DMFV models and definition of the benchmark scenarios}

In this section, we present the simplified models of top-flavoured dark matter introduced in $[13,14]$ that we will use throughout this analysis. We start by recapitulating the basics

\footnotetext{
${ }^{1}$ A leptonic DMFV model has been suggested in [15].
} 
of Dark Minimal Flavour Violation (DMFV), and then move on to briefly review the theoretical ingredients and phenomenological implications of the two models in which DM couples either to right- or left-handed top quarks. The experimental constraints identified in $[13,14]$ will guide us in deriving four viable benchmark scenarios for our analysis of LHC constraints and single-top signatures.

\subsection{The DMFV framework}

The original models of flavoured dark matter embedded the assumption of Minimal Flavour Violation (MFV) [16-20]: the SM Yukawa couplings were assumed to be the only source of flavour violation, and hence the flavour structure and phenomenology of those models was highly restricted. Subsequently, in order to allow for a richer flavour phenomenology, the concept of Dark Minimal Flavour Violation (DMFV) was introduced in [12]. In this framework, the coupling matrix $\lambda$ between the SM quarks and the fermionic DM field $\chi$, transforming as triplet under a new flavour symmetry $\mathrm{U}(3)_{\chi}$, is assumed to be the only new source of flavour violation beyond the SM Yukawa couplings. As a consequence, the three dark flavours $\chi_{i}$ are nearly degenerate, with a mass splitting generated only by corrections of the form $\eta \lambda^{\dagger} \lambda$. Here $\eta$ is a free parameter within the simplified model that would be determined by the choice of a UV completion. Thanks to the DMFV flavour symmetry, the lightest state in the dark sector is stable $[5,12]$ and is assumed to form the observed DM of the universe.

The interaction between $\chi$ and the SM quarks is mediated by a scalar $t$-channel mediator $\phi$ that carries QCD colour charge. Its electroweak quantum numbers determine whether the DM couples to right-handed up- or down-type quarks or to the left-handed quark doublets. We thus have two possible implementations of top-flavoured DM in the DMFV framework, with the lightest flavour of $\chi$ coupling either to the right- or the left-handed top quark. These two models, dubbed "right-handed" and "left-handed", are introduced next.

\section{$2.2 \quad$ Right-handed model}

In the right-handed model, the scalar mediator $\phi$ carries the same gauge quantum numbers as the right-handed up-type quarks. Thus the DM flavour triplet $\chi$ couples to the righthanded up-type quark triplet via the interaction term

$$
\mathcal{L}_{\mathrm{RH}} \supset-\lambda_{i j} \bar{u}_{R i} \chi_{j} \phi+\text { h.c. } .
$$

Here $\lambda$ is a $3 \times 3$ complex matrix that can be parametrised in terms of three diagonal couplings $D_{\lambda, i i}>0$, three flavour mixing angles $0 \leq \theta_{i j} \leq \pi / 4$ and three complex phases $0 \leq \delta_{i j}<2 \pi$ as follows:

$$
\begin{aligned}
\lambda & =U_{\lambda} D_{\lambda} \quad \text { with } \\
D_{\lambda} & =\operatorname{diag}\left(D_{\lambda, 11}, D_{\lambda, 22}, D_{\lambda, 33}\right), \\
U_{\lambda} & =\left(\begin{array}{ccc}
1 & 0 & 0 \\
0 & c_{23} & s_{23} e^{-i \delta_{23}} \\
0 & -s_{23} e^{i \delta_{23}} & c_{23}
\end{array}\right)\left(\begin{array}{ccc}
c_{13} & 0 & s_{13} e^{-i \delta_{13}} \\
0 & 1 & 0 \\
-s_{13} e^{i \delta_{13}} & 0 & c_{13}
\end{array}\right)\left(\begin{array}{ccc}
c_{12} & s_{12} e^{-i \delta_{12}} & 0 \\
-s_{12} e^{i \delta_{12}} & c_{12} & 0 \\
0 & 0 & 1
\end{array}\right),
\end{aligned}
$$


where $c_{i j}=\cos \theta_{i j}$ and $s_{i j}=\sin \theta_{i j}$. Note that the mixing angles $\theta_{i j}$ have been constrained to be at most $\pi / 4$ in order to ensure that the DM flavour $\chi_{i}$ couples predominantly to the $i$ th quark generation. We hence name $\chi_{3} \equiv \chi_{t}$ the top-flavoured state. As in [13], we consider $\chi_{t}$ to be the lightest dark flavour, i.e. DM is top-flavoured, ${ }^{2}$ which is favoured by the stringent upper limit on the WIMP-nucleus scattering cross-section from direct detection experiments.

\subsection{Left-handed model}

In the left-handed model, instead, the scalar mediator carries the same gauge quantum numbers as the left-handed quark doublets, i.e. it is introduced as an $\mathrm{SU}(2)_{L}$ doublet $\phi=\left(\phi_{u}, \phi_{d}\right)^{T}$. The coupling of the DM flavour triplet $\chi$ to the left-handed quarks then reads

$$
\begin{aligned}
\mathcal{L}_{\mathrm{LH}} & \supset-\lambda_{i j} \bar{q}_{L i} \chi_{j} \phi+\text { h.c. } \\
& =-\lambda_{i j} \bar{u}_{L i} \chi_{j} \phi_{u}-\tilde{\lambda}_{i j} \bar{d}_{L i} \chi_{j} \phi_{d}+\text { h.c. }
\end{aligned}
$$

Here, $\lambda$ is parametrised as in $(2.2)-(2.4)$, and

$$
\tilde{\lambda}=V_{\mathrm{CKM}}^{\dagger} \lambda
$$

accounts for the CKM misalignment between left-handed up- and down-type quarks. Again we assume $\chi_{3} \equiv \chi_{t}$ to be the lightest dark flavour and refer to it as top-flavoured DM. It couples to the SM top quark via $\phi_{u}$ and to the SM bottom quark via $\phi_{d}$.

\subsection{Phenomenology and benchmark scenarios}

We now briefly summarise the phenomenology of both models, as analysed in detail in [13, 14]. The constraints on their parameter space will guide us in our choice of four benchmark scenarios, on which we will focus our subsequent study of LHC signatures.

In the right-handed $(\mathrm{RH})$ model, the most relevant constraint from flavour physics is due to the data on neutral $D$ meson mixing, requiring the mixing angle $\theta_{12}$ to be small unless the first two generation couplings, $D_{\lambda, 11}$ and $D_{\lambda, 22}$ are nearly degenerate. The non-observation of WIMP DM in direct detection liquid xenon experiments requires a significant cancellation between tree-level and $Z$-penguin contributions to the DM-nucleon scattering cross-section, requiring in particular a non-zero mixing angle $\theta_{13}$. Concerning the early universe phenomenology, different scenarios can be distinguished, depending on the lifetime of the heavier dark flavours $\chi_{1,2}$. The thermal freeze-out condition depends on the number of dark flavours present at the time the dark sector decoupled from the visible sector. In [13] two benchmark cases were investigated: quasi-degenerate freeze-out (QDF), in which the decay of the heavy flavours takes place after the DM freeze-out, so that all three flavours contribute to the effective annihilation cross-section, and single flavour freezeout (SFF), in which the heavier flavours have decayed into $\chi_{t}$ already in the equilibrium phase. These two cases are distinguished mainly by the mass splitting between the different

\footnotetext{
${ }^{2}$ The possibility of charm-flavoured DM has been considered in [21].
} 


\begin{tabular}{|l|lll|}
\hline & DM mass & couplings & mixing angles \\
\hline RH-SFF & $m_{\chi}=200 \mathrm{GeV}$ & $D_{\lambda, 11}=D_{\lambda, 22}$ & $\sin \theta_{13}=0.25$ \\
& & $D_{\lambda, 33}=D_{\lambda, 11}+1.0$ & $\theta_{12}=\theta_{23}=0$ \\
\hline RH-QDF & $m_{\chi}=150 \mathrm{GeV}$ & $D_{\lambda, 11}=D_{\lambda, 22}$ & $\sin \theta_{13}=0.2$ \\
& & $D_{\lambda, 33}=D_{\lambda, 11}+0.2$ & $\theta_{12}=\theta_{23}=0$ \\
\hline LH-QDF1 & $m_{\chi}=150 \mathrm{GeV}$ & $D_{\lambda, 11}=D_{\lambda, 22}$ & $\sin \theta_{13}=0.1$ \\
& & $D_{\lambda, 33}=D_{\lambda, 11}+0.1$ & $\theta_{12}=\theta_{23}=0$ \\
\hline LH-QDF2 & $m_{\chi}=450 \mathrm{GeV}$ & $D_{\lambda, 11}=D_{\lambda, 22}$ & $\sin \theta_{13}=0.2$ \\
& & $D_{\lambda, 33}=D_{\lambda, 11}+0.2$ & $\theta_{12}=\theta_{23}=0$ \\
\hline
\end{tabular}

Table 1. Definition of benchmark scenarios. In all cases the mediator mass $m_{\phi}$ and the coupling $D_{\lambda, 11}$ are free parameters, while the complex phases $\delta_{i j}$ are set to zero.

flavours $\chi_{i}$, which in the DMFV framework is generated by the flavour non-universality in the couplings $D_{\lambda, i i}$ : while the QDF scenario requires a relative mass splitting of $1 \%$ or less, in the SFF scenario a mass splitting of about $10 \%$ is assumed. ${ }^{3}$

Altogether, the combination of the various constraints leads us to the identification of two interesting benchmark scenarios for the right-handed model, summarised in table 1. RH-SFF describes a typical set of parameters in the single-flavour freeze-out scenario, while RH-QDF picks a viable benchmark for the quasi-degenerate case.

The left-handed (LH) model is more stringently constrained by flavour physics, due to its couplings to both up and down quark sectors. Consequently, the constraints from neutral kaon, $B_{d, s}$ and $D$ meson oscillations come into play. The unavoidable CKM misalignment between the couplings $\lambda$ and $\tilde{\lambda}$ requires the first two generations to be quasi-degenerate, i.e. $D_{\lambda, 11} \simeq D_{\lambda, 22}$. In this limit, the mixing angle $\theta_{12}$ becomes unphysical. In addition, the data from $B_{d, s}$ meson mixing require the mixing angles $\theta_{13}$ and $\theta_{23}$ to be small unless the third generation, $\chi_{t}$, is quasi-degenerate with the first two. At the same time, as in the right-handed model, the required cancellation in the WIMP-nucleus scattering cross-section bounded by direct detection experiments demands a non-vanishing mixing angle $\theta_{13}$. The combination of direct detection and flavour physics constraints thus excludes the singleflavour freeze-out scenario in the left-handed model. We hence choose two benchmark scenarios within the quasi-degenerate freeze-out case, LH-QDF1 and LH-QDF2, that differ most notably in the DM mass.

\section{LHC phenomenology}

At the LHC, the scalar mediator $\phi$ can be pair-produced by QCD interactions. It can also be pair- or singly produced through its interactions with the DM triplet $\chi$ and the SM quarks, parametrised by the matrix $\lambda$. In this section, we first perform a general study of

\footnotetext{
${ }^{3}$ Note that even in the single-flavour freeze-out scenario, the decay of the heavy flavours is irrelevant for the purpose of the present paper as the visible decay products are too soft to be identified in the LHC searches discussed below. The distinctive LHC signatures of the heavier DM flavours decaying into the lightest state have been discussed in [22].
} 

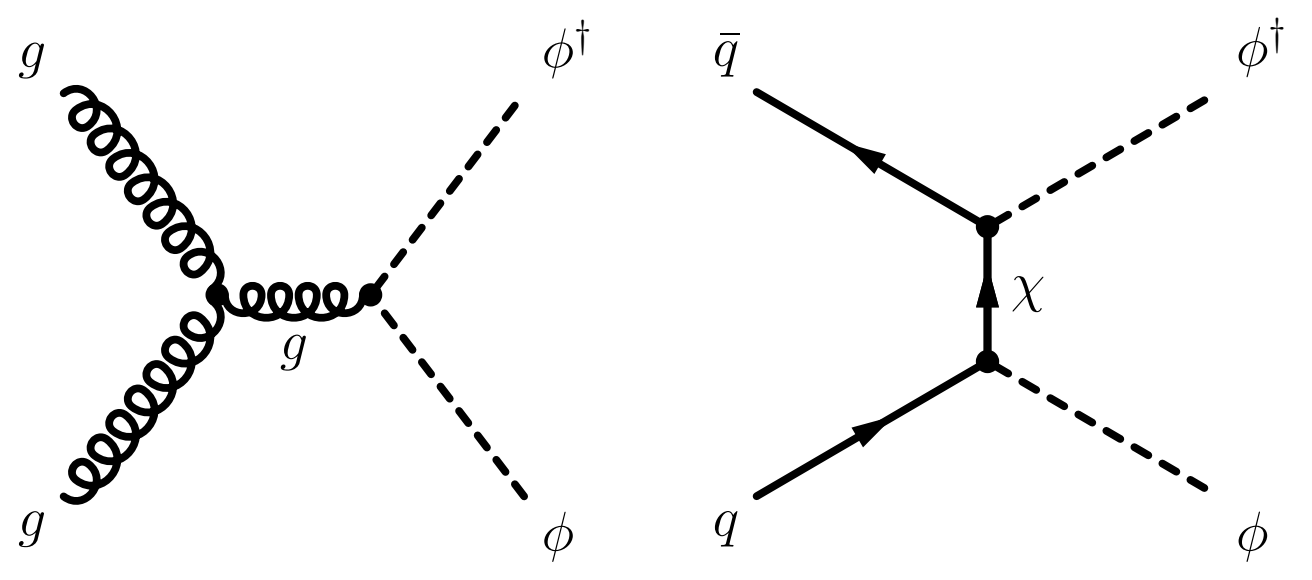

Figure 1. Representative Feynman diagrams for the production of mediator pairs.

the final states resulting from the pair production of mediators. After that, we concentrate on the final states featuring a single top quark, which can result either from pair or single production of mediators. For all the considered final states, we study the dependence of the expected production rates on the parameters of the model for the benchmark cases introduced in the previous section.

\subsection{Pair production of mediators}

The mediator $\phi$ can be pair-produced either through $s$-channel or through $t$-channels processes. Representative diagrams for the two processes are shown in figure 1 . The crosssection for $s$-channel production, a pure QCD process, is independent of the parameters of the model, except for the mediator mass. The cross-section for $t$-channel production depends instead on all parameters, in particular it has a quadratic dependence on the product of the two relevant couplings.

The total production cross-sections for the four benchmarks are shown in figure 2 as a function of the coupling $D_{\lambda, 11}$. Only one type of mediator $\phi$ is produced in the RH benchmarks. At low $D_{\lambda, 11}$ the $t$-channel diagrams slightly decrease the total cross-section through a negative interference with the $s$-channel diagrams. With increasing $D_{\lambda, 11}$ the $t$-channel becomes dominant, and it overtakes the $s$-channel for a value of $D_{\lambda, 11}$ which depends on $D_{\lambda, 33}$, due to the non-zero $\theta_{13}$ mixing angle. In the case of the LH benchmarks, three configurations for the pair production of mediators are relevant: $\phi_{u} \phi_{u}, \phi_{d} \phi_{d}$, and $\phi_{u} \phi_{d}$. For the first two, the dependence on $D_{\lambda, 11}$ looks like the one discussed for the RH benchmarks, whereas $\phi_{u} \phi_{d}$ is a pure $t$-channel process and grows with $D_{\lambda, 11}$ over the full considered $D_{\lambda, 11}$ range. The cross-section of $\phi_{u} \phi_{u}$ for LH-QDF1 is very similar to the one for RH-QDF, which has comparable parameters. The cross-sections of all three processes are larger for LH-QDF1 than for LH-QDF2 at high $D_{\lambda, 11}$ because of the larger value of the $\chi$ mass exchanged in the $t$-channel for the latter benchmark.

The cross-sections need to be convoluted with the branching fractions of the mediators into the different quark flavours to obtain the cross-sections for the final states of interest. The branching ratios (BR) are determined by the squares of the relative values of the 

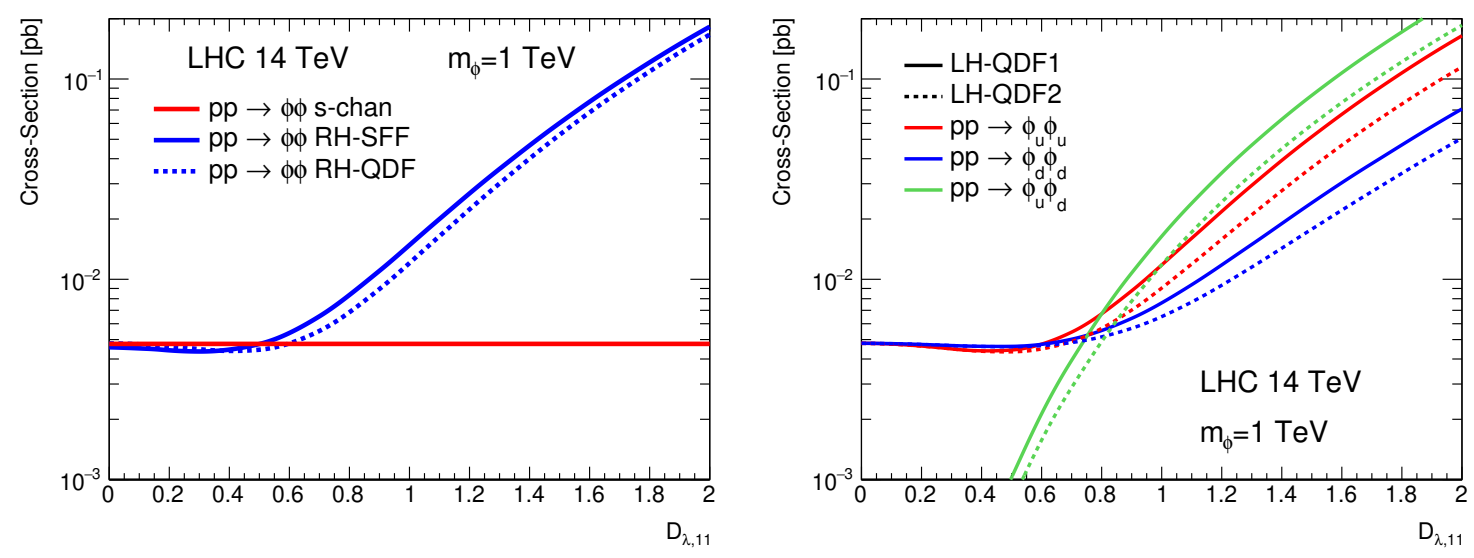

Figure 2. Cross-section for resonant production of two $\phi$ mediators as a function of $D_{\lambda, 11}$ at a $14 \mathrm{TeV}$ LHC. The value of $m_{\phi}$ is fixed at $1 \mathrm{TeV}$. Left: RH-SFF (solid line) and RH-QDF1 (dashed line) benchmarks. Right: cross-section for three configurations: $\phi_{u} \phi_{u}, \phi_{d} \phi_{d}, \phi_{u} \phi_{d}$ for benchmarks LH-QDF1 (solid line) and LH-QDF2 (dashed line).

couplings $D_{\lambda, 11}$ and $D_{\lambda, 33}$. In the case of equal couplings $D_{\lambda, 11}=D_{\lambda, 22}=D_{\lambda, 33}$, the mediator would have $33 \%$ BR into 3rd generation quarks and $66 \%$ into quarks of the first two generations.

Since the right-handed model features only one type of mediator, coupling to up-type quarks, we have three final states: $j j+E_{\mathrm{T}}^{\text {miss }}$ (henceforth shortened to $j j$ ), $t \bar{t}+E_{\mathrm{T}}^{\text {miss }}(t \bar{t})$, and $t j+E_{\mathrm{T}}^{\text {miss }}(t j)$ where $j$ is a quark from the first two generations. An interesting configuration is realised for $D_{\lambda, 11}=D_{\lambda, 22}=0.5 \times D_{\lambda, 33}$. This case yields the most favourable situation for the $t j$ final state which has a $50 \% \mathrm{BR}$, as compared to $25 \%$ each for $j j$ and $t \bar{t}$. The value of $D_{\lambda, 11}$ for which this happens for each of the benchmarks ultimately determines the phenomenology.

The branching fractions for the three final states in mediator pair production are shown in figure 3. In the RH-SFF benchmark (left), with $D_{\lambda, 33}=D_{\lambda, 11}+1, t \bar{t}$ is dominant up to $D_{\lambda, 11}=0.8$, where $t j$ takes over. For RH-QDF, with approximately balanced $D_{\lambda, 11}$ and $D_{\lambda, 33}$, the switch between $t \bar{t}$ and $t j$ happens already for $D_{\lambda, 11}=0.2$. Above this value, $\operatorname{BR}(t j)$ is approximately flat at $\sim 50 \%$. The non-zero value of $\theta_{13}$ is visible in the fact that the $t \bar{t}$ signature does not saturate the production for $D_{\lambda, 11} \sim 0$. This behaviour translates directly into the cross-sections for the three signatures, shown in figure 4.

Due to the $\mathrm{SU}(2)_{L}$ structure, the situation is more complex in the case of the LH benchmarks. In this case we are dealing with three different production processes, $\phi_{u} \phi_{u}$, $\phi_{d} \phi_{d}, \phi_{u} \phi_{d}$, and each of them gives rise to different final states combinations. For the $\phi_{u} \phi_{u}$ process, the allowed final states of interest are $j j, t \bar{t}$ and $t j$, and the BR pattern is very similar to the one shown in the right panel of figure 3. For $\phi_{d} \phi_{d}$ and $\phi_{u} \phi_{d}$, different channels are open, and the BRs are shown in figure 5. The $j j$ final state is allowed in all three production processes, $t j$ in two, and the purely third generation signatures only in one. The final cross-sections for the different signatures combining the three production processes are shown in figure 6 . The coupling dependence of the five relevant signatures is very similar for the two benchmarks, with the cross-sections for LH-QDF2 somewhat 

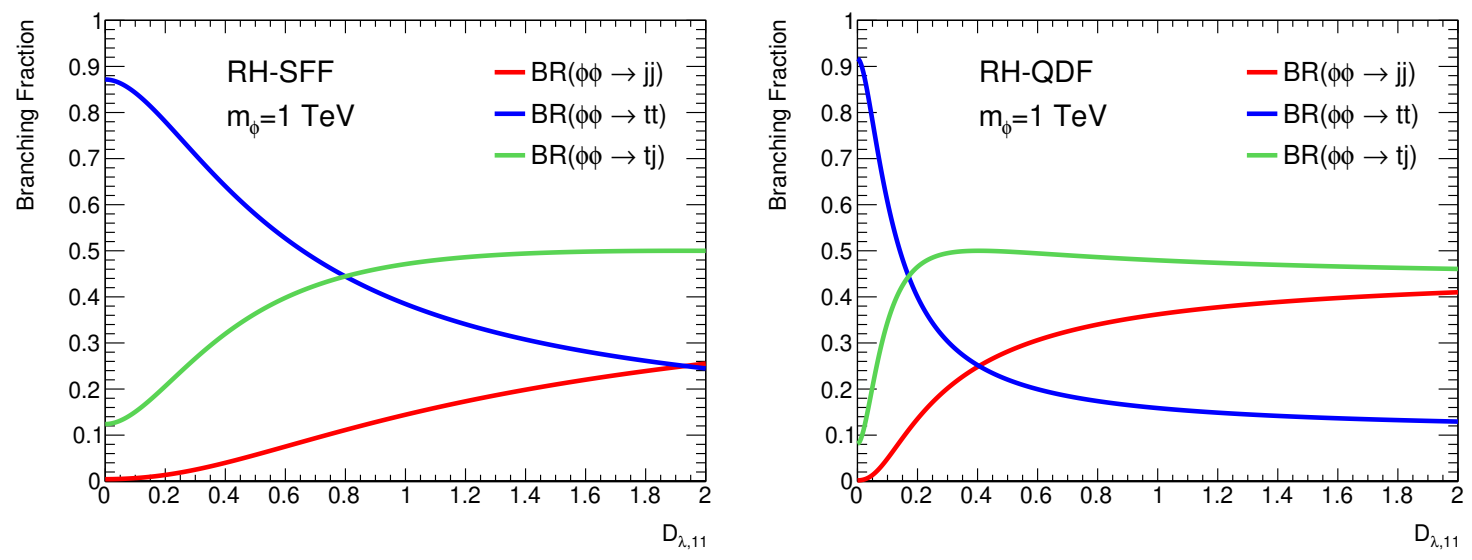

Figure 3. Branching fractions for three final states: $j j+E_{\mathrm{T}}^{\mathrm{miss}}, t t+E_{\mathrm{T}}^{\mathrm{miss}}$ and $t j+E_{\mathrm{T}}^{\mathrm{miss}}$ as a function of the coupling $D_{\lambda, 11}$ for the two RH benchmarks. Left: RH-SFF benchmark: right: RH-QDF benchmark.
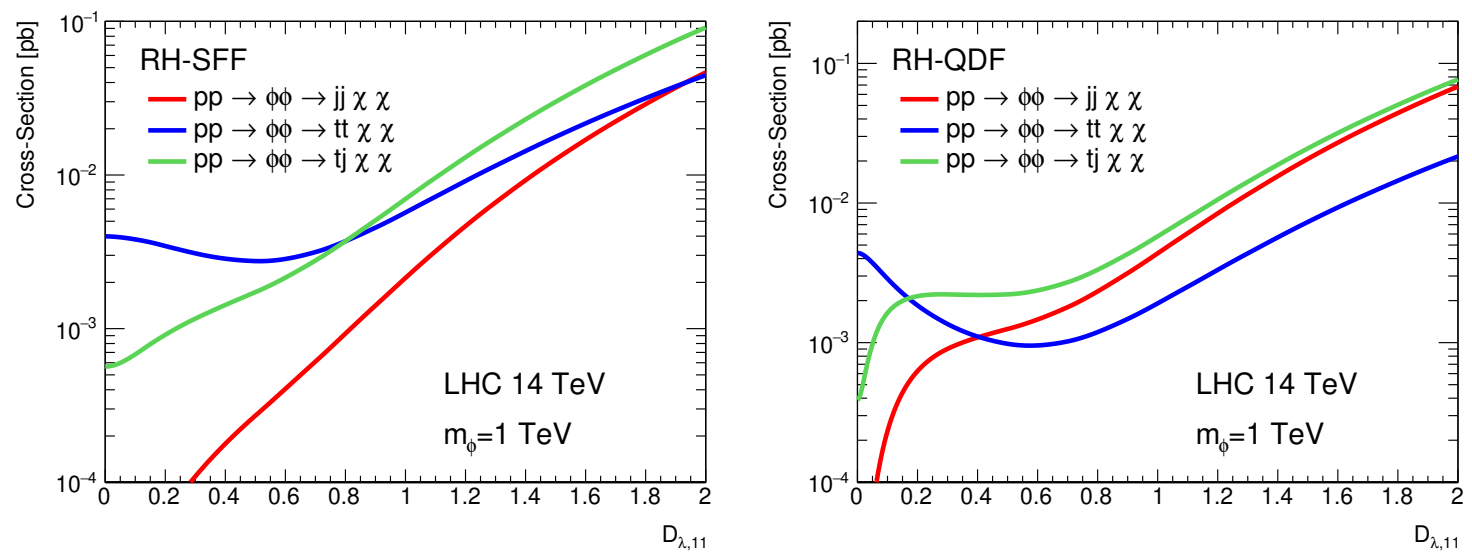

Figure 4. Production cross-section for the different final states resulting from pair production of mediators at a $14 \mathrm{TeV}$ LHC for the two RH benchmarks as a function of $D_{\lambda, 11}$. Left: RH-SFF, right: RH-QDF. The assumed mediator mass is $1 \mathrm{TeV}$.

lower. The $j j$ final state is dominant starting from $D_{\lambda, 11}=0.2-0.3$, with $t j$ being not far below. In the region where $j j$ and $t \bar{t}$ are of similar size, the $t j$ signature becomes dominant. For $D_{\lambda, 11}$ close to zero, $t \bar{t}$ and $b \bar{b}$ have the largest cross-sections.

\subsection{Single top final states}

Signatures including a single top quark can be produced in two ways in DMFV models: either through the on-shell production of a pair of mediators, one of which decays into a top quark and a dark matter particle, as already introduced in section 3.1, or through the production of a single mediator, either accompanied by a top quark or by a dark matter particle.

We classify in the following the possible single top signatures at the LHC. We neglect signatures where the top quark is produced in the decay of a mediator and the additional 

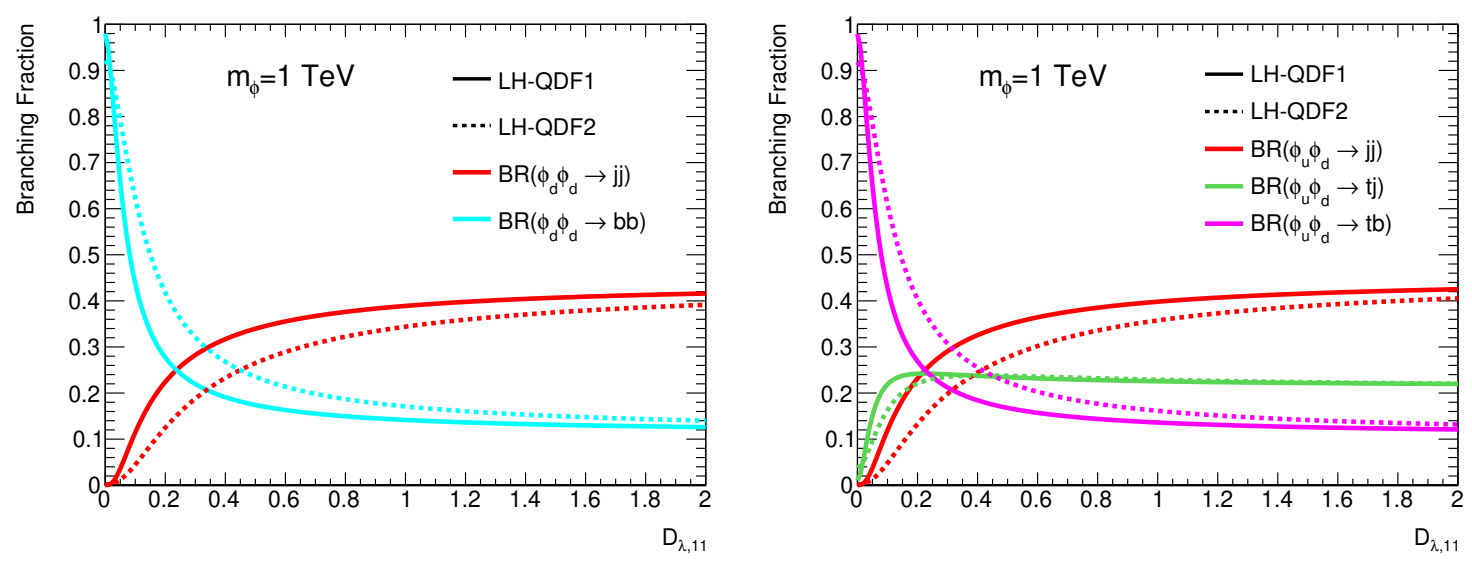

Figure 5. Branching fractions for the allowed final states respectively for $\phi_{d} \phi_{d}$ (left) and $\phi_{u} \phi_{d}$ (right) production as a function of $D_{\lambda, 11}$. The solid (dashed) lines are for LH-QDF1 (LH-QDF2).
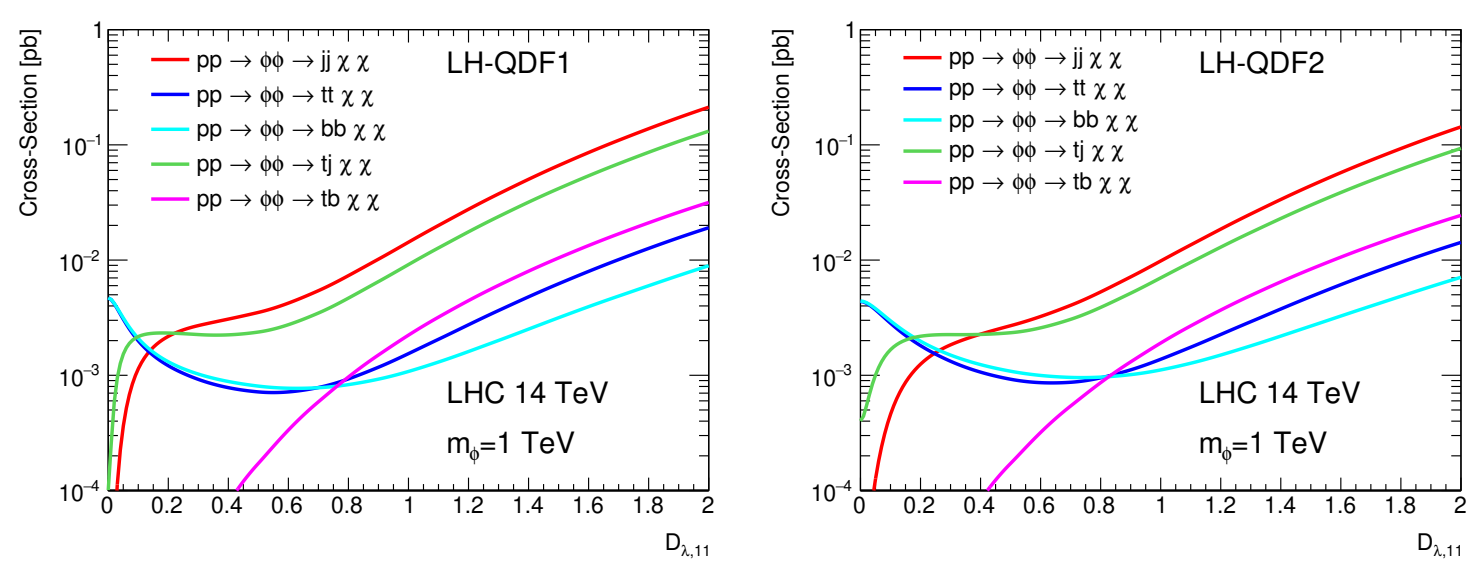

Figure 6. Production cross-section for the different final states resulting from pair production of mediators at a $14 \mathrm{TeV}$ LHC for the two LH benchmarks as a function of $D_{\lambda, 11}$. Left: LH-QDF1, right: LH-QDF2. The assumed mediator mass is $1 \mathrm{TeV}$.

light or b-quark is produced in QCD radiation. The latter represent different final-state topologies as the additional quark is not produced in the two-body decay of a heavy particle. We identify the following three final states:

- $t+E_{\mathrm{T}}^{\mathrm{miss}}$

- $t+\bar{q}+E_{\mathrm{T}}^{\mathrm{miss}}$, with $q=(u, d, s, c)$

- $t+\bar{b}+E_{\mathrm{T}}^{\mathrm{miss}}$.

In this section, we analyse the origin of each of the signatures above in the right-handed up model and in the left-handed model. In section 5, we will then develop an LHC analysis strategy for each of the signatures and evaluate the reach in parameter space for the full LHC statistics and for the HL-LHC run. 


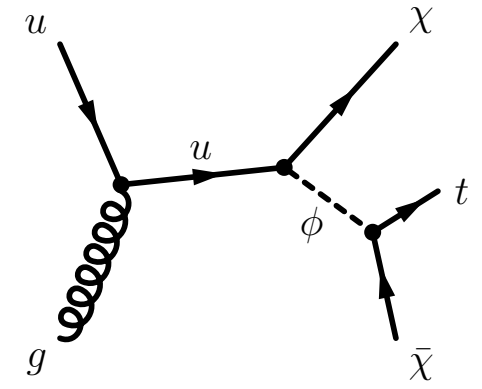

(a)

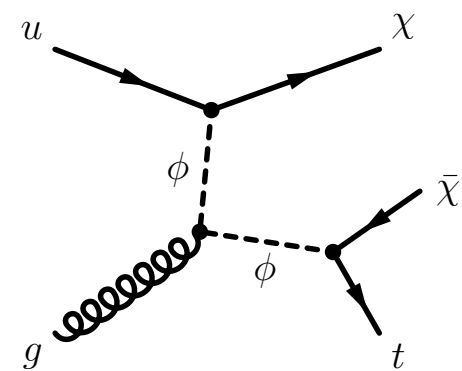

(b)

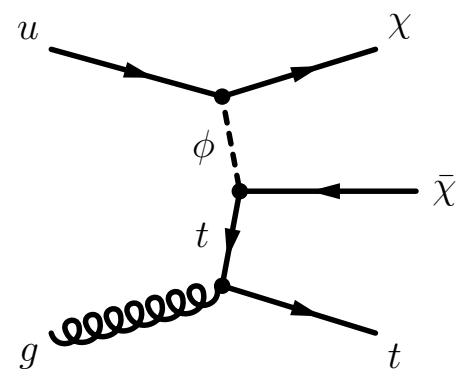

(c)

Figure 7. Representative Feynman diagrams for the production of two dark matter particles $\chi$ in association with a single top quark.

$\boldsymbol{t}+\boldsymbol{E}_{\mathbf{T}}^{\text {miss }}$. Representative Feynman diagrams leading to the $t+E_{\mathrm{T}}^{\text {miss }}$ final state are shown in figure 7. The initial state is always a quark and a gluon. For the first two diagrams on the left, (a) and (b), the top quark is produced in the decay of an on-shell mediator, whereas diagram (c) features a mediator exchanged in the $t$-channel.

The signature consisting in a single top quark recoiling against two dark matter particles is frequently dubbed mono-top signature [23, 24], and is similar to the one exploited in the ATLAS and CMS searches documented in [25, 26]. The signals considered in these studies, however, have a final state kinematics which is different from the one of the production processes shown in figure 7 . Therefore, rather than attempting a recasting, we will develop a dedicated analysis strategy optimised for our benchmarks and based on the semileptonic decay of the top quark.

$\boldsymbol{t}+\overline{\boldsymbol{q}}+\boldsymbol{E}_{\mathbf{T}}^{\text {miss }} . \quad$ Representative Feynman diagrams leading to the final state $t+\bar{q}+E_{\mathrm{T}}^{\text {miss }}$ are shown in figure 8. The diagram in subfigure (a) depicts the pure QCD production of two on-shell mediators and is equivalent to the non-MFV SUSY production of two differentflavour quarks [27, 28]. Process (b) features the pair production of two mediators with a dark matter particle exchanged in the $t$-channel. The final states with a $d$ or $s$ quark arise only in the LH model. For the diagram of subfigure (c), the mediator is radiated from a $t$-quark leg in $t \bar{t}$ production. The experimental reach for the process in subfigure (a) was studied in [29] in the framework of a non-MFV simplified SUSY model, but it has not yet been the subject of an analysis by the LHC experiments.

Building on the analysis of [29], we present a re-optimisation of the selections based on a detailed simulation of all the processes contributing to the addressed final state.

$\boldsymbol{t}+\overline{\boldsymbol{b}}+\boldsymbol{E}_{\mathrm{T}}^{\mathrm{miss}}$. This final state is only produced in the left-handed version of the model. A representative Feynman diagram leading to the final state $t+\bar{b}+E_{\mathrm{T}}^{\text {miss }}$ is shown in figure 9 . Since the $b$-jet is identifiable experimentally, no $s$-channel production mechanism is available, differently from the $t+\bar{q}+E_{\mathrm{T}}^{\text {miss }}$ channel.

The $t+\bar{b}+E_{\mathrm{T}}^{\text {miss }}$ signature is specific to this model and does not arise from pairproduction of SUSY squarks. It was studied by the ATLAS experiment [30], however, in the framework of the searches for cascade decays of the sbottom quark, targeting SUSY 


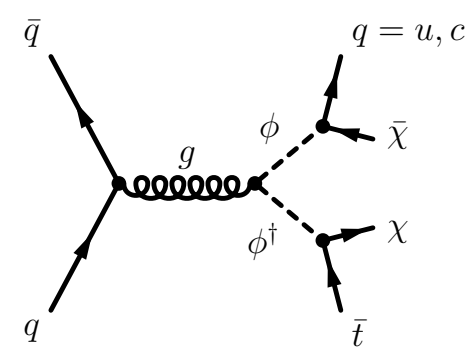

(a)

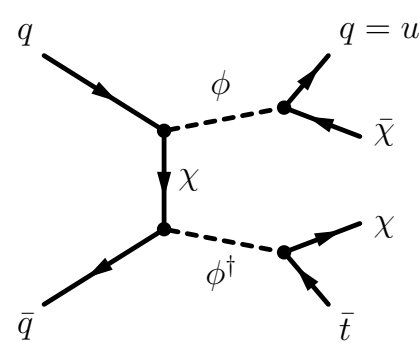

(b)

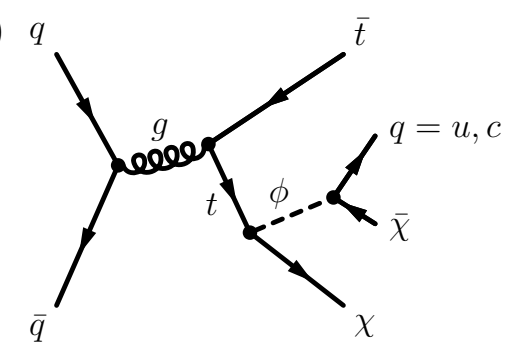

(c)

Figure 8. Representative Feynman diagrams for the production of DM in association with a light jet and a top quark.

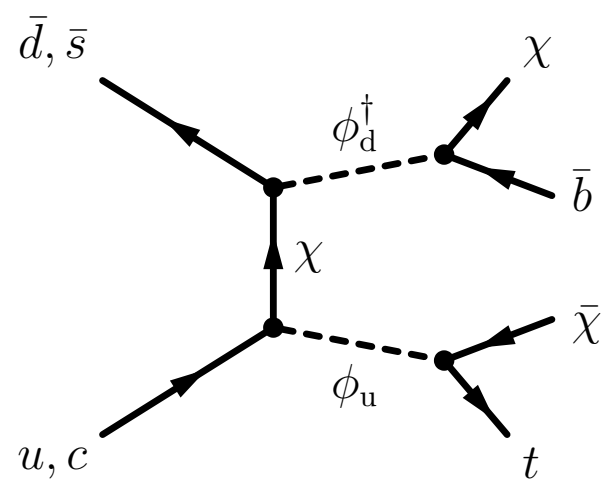

Figure 9. Feynman diagram for the production of DM in association with a top and a $b$-quark.

models with compressed mass spectra. The addressed kinematic is very different from the one for the DMFV $t+\bar{b}+E_{\mathrm{T}}^{\text {miss }}$ final state. We will therefore develop an independent analysis strategy.

The cross-sections for the three processes discussed above are shown in figure 10 for the four benchmarks. In what follows, we will use the shorthand notation monotop, $t j$ and $t b$, respectively, for the three signatures.

For $t j$ and $t b$, the full production cross-section, shown as a solid line, is compared to the one from mediator pair production, shown as a dashed line. Both cross-sections are dominated by the doubly-resonant component, and the discussion in section 3.1 applies.

The monotop signature is dominant over the whole parameter range considered for the RH-SFF benchmark. The three remaining benchmarks have a similar pattern, with monotop dominating $t j$ down to $D_{\lambda, 11}=0.1-0.2$. The monotop cross-sections are similar for RH-QDF and LH-QDF1, while they are a factor 2-3 lower for LH-QDF2, due to the much larger mass of $\chi$.

Last but not least, the $t b$ signature in the LH model has a significantly smaller crosssection than both monotop and $t j$ over the full range of parameters considered.

\section{Recast of LHC limits for mediator pair production}

We consider the four benchmarks described in the previous section and we explore the existing LHC bounds on these two models from the on-shell productions of two coloured 

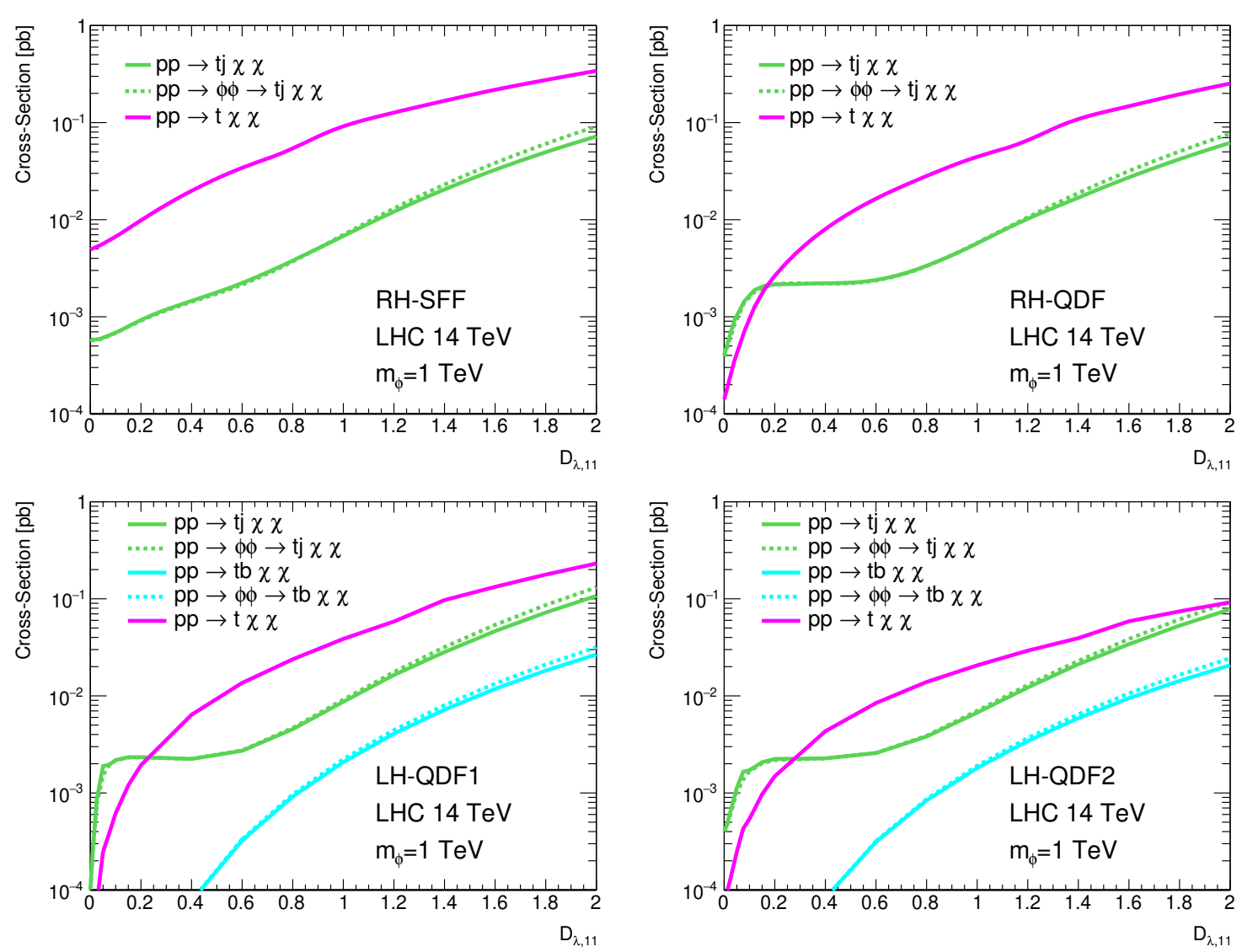

Figure 10. Production cross-section for the single top signatures in $14 \mathrm{TeV}$ proton-proton collisions for the four benchmarks, as a function of $D_{\lambda, 11}$. For the $t j$ and $t b$ final states both the value for the full cross-section and the one through resonant production of mediator pairs is shown. The value of $m_{\Phi}$ is fixed at $1 \mathrm{TeV}$.

mediators $\phi$ which in turn decay into a quark and a dark matter particle $\chi$, which is the same final state studied by SUSY squark searches.

The recast of published searches for the production of two squarks relies on the assumption that the selection efficiency of each of the considered analyses is the same for our model and for the simplified SUSY ones used in the experimental analysis, assuming the mass values such that $m_{\tilde{q}}=m_{\phi}$ and $m_{\tilde{\chi}_{1}^{0}}=m_{\chi}$.

In all four benchmarks, for each mass of the mediator $\phi$ and each $D_{\lambda, 11}$ value the cross-section for the process

$$
p p \rightarrow \phi \phi^{\dagger}
$$

is calculated for the relevant LHC centre-of-mass energy, as well the branching fractions of $\phi \rightarrow q \chi_{q}$, where q runs over all the quark flavours relevant for each benchmark, as discussed in section 3.1. Based on the production cross-sections and branching fractions, the total cross-section for each of the considered three final states is calculated.

The published LHC SUSY searches are in the framework of MFV models. Therefore, the experimental SUSY results relevant for this study are the searches for pair productions 
of squarks of the first two generations, of stop squarks, and of sbottom squarks, addressing the final states $j j, t \bar{t}$ and $b \bar{b}$ of section 3.1. At the time of writing, only a limited set of relevant analyses based on the full LHC Run 2 statistics of $\sim 140 \mathrm{fb}^{-1}$ have been published [31-34]. A necessary condition for the present study is the availability in tabular form of the limits on the SUSY cross-section over a broad range of squark masses, extending down to $\sim 500 \mathrm{GeV}$ for the values of $m_{\tilde{q}}$ assumed by our benchmark models. The CMS study published in [31] presents a search for strongly produced SUSY in final states with multiple hadronic jets and $E_{\mathrm{T}}^{\text {miss }}$ based on a statistics of $137 \mathrm{fb}^{-1}$. The direct production of squarks in simplified models resulting in all of the three final states of interest, $j j+E_{\mathrm{T}}^{\text {miss }}$, $t \bar{t}+E_{\mathrm{T}}^{\mathrm{miss}}$ and $b \bar{b}+E_{\mathrm{T}}^{\mathrm{miss}}$, is explicitly addressed in the paper, and tables of cross-section limits on very fine grids in $\left(m_{\tilde{q}}, m_{\tilde{\chi}_{1}^{0}}\right)$ are provided for masses of squarks of the first two generations between 500 and $2000 \mathrm{GeV}$, going down to even lower masses for stop and sbottom. We base our recasting study on this work. For stop final states, a dedicated ATLAS analysis is also available [34] based on $140 \mathrm{fb}^{-1}$, addressing as well a fully hadronic final state. They exclude a stop mass of approximately $1250 \mathrm{GeV}$ for a massless $\tilde{\chi}_{1}^{0}$, as compared to the $1150 \mathrm{GeV}$ for the CMS analysis, but the exclusion tables provided cover a smaller range of stop masses, up to $1450 \mathrm{GeV}$, and they have a coarser granularity. We therefore use the results of [31] for $t \bar{t}+E_{\mathrm{T}}^{\text {miss }}$ as well, although the limits are not the best available ones. Alternative CMS analyses [32,33] exclude a stop mass of $1200 \mathrm{GeV}$, but they do not provide results in tabular form at the time of writing.

For each $D_{\lambda, 11}$ value considered, the expected cross-section for each signature as a function of $m_{\phi}$ is compared to the mass-dependent excluded cross-section from [31], and the mass value where the two curves cross is taken as excluded mass for that configuration of couplings. The results of the exercise are shown in figure 11. For the RH-SFF benchmark, where the $D_{\lambda, 33}$ coupling is much larger than $D_{\lambda, 11}$, the $t \bar{t}+E_{\mathrm{T}}^{\text {miss }}$ signature is dominant over the whole considered parameter space, and $m_{\phi}$ lower than $900 \mathrm{GeV}$ are excluded for all values of $D_{\lambda, 11}$. For the RH-QDF benchmark, where the first and third-generation couplings have a comparable value, $t \bar{t}+E_{\mathrm{T}}^{\text {miss }}$ dominates at low $D_{\lambda, 11}$ and $j j+E_{\mathrm{T}}^{\text {miss }}$ at high $D_{\lambda, 11}$, and values of $m_{\phi}$ up to $650 \mathrm{GeV}$ are excluded. Concerning LH-QDF1, which has a similar pattern of couplings but the additional contribution of down-type quarks, $b \bar{b}+E_{\mathrm{T}}^{\text {miss }}$ is dominant at low $D_{\lambda, 11}$, with very similar power as $t \bar{t}+E_{\mathrm{T}}^{\text {miss }}$, but $j j+E_{\mathrm{T}}^{\text {miss }}$ again provides the best exclusion for high $D_{\lambda, 11}$. The interplay of $b \bar{b}+E_{\mathrm{T}}^{\text {miss }}$ and $j j+E_{\mathrm{T}}^{\text {miss }}$ brings the excluded value of $m_{\phi}$ to $800 \mathrm{GeV}$. A similar pattern is observed for LH-QDF2, but the very high value of $m_{\chi}$ strongly reduces the analysis acceptance for low mediator masses, leaving a large interval of $D_{\lambda, 11}$ for which no value of $m_{\phi}$ is excluded. Dedicated analyses targeting models with small mass differences between squark and neutralino are needed to improve the sensitivity in that area.

The orange dash-dotted lines in figure 11 indicate for which set of parameters the correct relic abundance is obtained, assuming thermal freeze-out. To the left of the line, an additional DM component is required to explain the observed DM density. To the right of the line, thermal freeze-out leads to a too large DM density, an extension of the model would hence be required to avoid an overclosing universe. The minimal model with the correct relic abundance is excluded by $j j+E_{\mathrm{T}}^{\text {miss }}$ for the LH-QDF benchmark, and by 

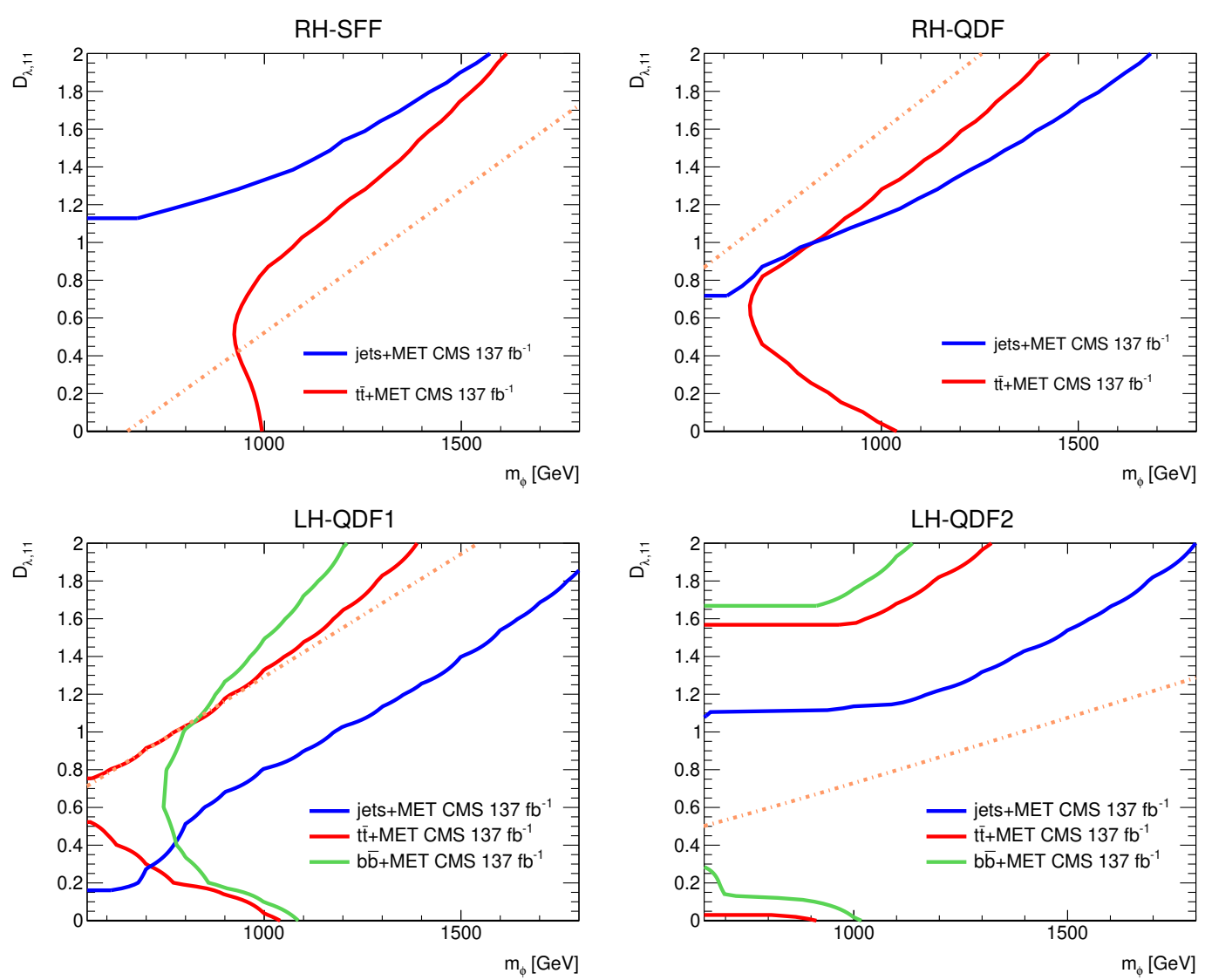

Figure 11. Excluded areas in the $\left(m_{\phi}, D_{\lambda, 11}\right)$ plane based on the analyses of the CMS collaboration on the full Run 2 statistics $13 \mathrm{TeV}$ LHC data for the four benchmarks models proposed in this paper. The excluded areas are on the left of the curves. The orange dash-dotted lines indicate for which set of parameters the correct relic abundance is obtained.

both $j j+E_{\mathrm{T}}^{\text {miss }}$ and $t \bar{t}+E_{\mathrm{T}}^{\text {miss }}$ for the RH-QDF benchmark. The simple thermal freeze-out assumption is however still viable in RH-SFF, due to the large splitting between the DM couplings to the different quark flavours, and in LH-QDF2, due to the large DM mass $m_{\chi}=450 \mathrm{GeV}$.

\section{Detailed analyses of single-top signatures}

For each of the three single-top signatures addressed in section 3, we perform a detailed study of the LHC prospects, focusing in each case on the semileptonic decay of the top quark. The final states of interest will then always include an isolated lepton $(e, \mu)$, a hadronic jet tagged as the result of the fragmentation of a $b$-quark, and $E_{\mathrm{T}}^{\text {miss }}$ both from the neutrino from the top decay and from the production of two $\chi$ particles which escape the detector undetected. We will show in the following how, for each of the considered signatures, kinematic cuts can be defined to reduce to a manageable level the very large backgrounds from $t \bar{t}$ and $W+$ jets productions. 


\subsection{Monte Carlo simulation}

All the samples are generated for a centre-of-mass energy of the LHC of $14 \mathrm{TeV}$. The signal samples are generated at LO using the DMFV UFO model [35] implementations provided in $[13,14]$. Parton-level events are generated with MadGraph5_aMC@NLO [36], employing NNPDF3.0 parton distribution functions (PDFs) [37], and showered with PYTHIA 8.2 [38].

For each of the three benchmarks, we generate a string of event samples with mediator mass variable between $400 \mathrm{GeV}$ and $3 \mathrm{TeV}$ and fixed values of the couplings $D_{\lambda, 11}=D_{\lambda, 22}=$ 0.4. A grid of event samples with fixed mediator mass $m_{\phi}=1 \mathrm{TeV}$ and $D_{\lambda, 11}=D_{\lambda, 22}$ variable between 0 and 2 is generated in order to evaluate the dependence of the experimental acceptance on the different sample compositions over the $\left(D_{\lambda, 11}, D_{\lambda, 22}\right)$ plane.

The proposed analyses address final states with a single isolated lepton, therefore all of the Standard Model processes featuring a lepton in the final state are considered for the background evaluation.

Backgrounds either with fake electrons from jet misidentification or with real nonisolated leptons from the decays of heavy-flavour hadrons are not considered in this study. An understanding of detector effects beyond the scope of this study would be needed for a reliable estimate. We estimate, based on the results of the ATLAS experiment, these backgrounds not to exceed around $15 \%$ of the total background surviving our selections.

Backgrounds from $t \bar{t}$ [39], $t W$ [40], $W W, W Z$ and $Z Z$ production [41, 42] are generated at next-to-leading order (NLO) with POWHEG BOX [43]. Samples of jets $+Z$ and jets $+W$ events are generated at LO with MadGraph5_aMC@NLO and considering up to three jets for the matrix element calculation. The $t \bar{t} V$ backgrounds with $V=W, Z$ are also simulated with MadGraph5_aMC@NLO at LO with a multiplicity of up to two jets, and the $t Z$ and $t W Z$ backgrounds at LO. All partonic events are showered with PYTHIA 8.2. The samples produced with POWHEG BOX are normalised to the NLO cross section given by the generator, except $t \bar{t}$ which is normalised to the cross section obtained at next-to-next-to-leading order (NNLO) plus next-to-next-to-leading logarithmic accuracy $[44,45]$. The jets $+W / Z$ samples are normalised to the known NNLO cross sections [46, 47], while the $t \bar{t} V$ samples are normalised to the NLO cross-section as calculated by MadGraph5_aMC@NLO.

The analysis is performed based on the following objects: leptons $(e, \mu)$, hadronic jets and $E_{\mathrm{T}}^{\text {miss }}$. Stable leptons produced in the decays of real $W$ and $Z$ and isolated from hadronic jets are considered in the analysis. Jets are built out of the momenta of all the stable particles depositing energy in the calorimeter except muons using an anti- $k_{t}$ algorithm [48] with a parameter $R=0.4$, as implemented in FastJet [49]. Jets originating from the hadronisation of bottom-quarks ( $b$-jets) are experimentally tagged with high efficiency (b-tagged jets). The $\vec{p}_{\mathrm{T}}^{\text {miss }}$ vector with magnitude $E_{\mathrm{T}}^{\text {miss }}$ is built out of the transverse momenta of all the invisible particles in the event.

The experimental effects are simulated by smearing the momenta of the analysis objects and by applying efficiency factors where applicable. The smearing and efficiency functions used to this purpose are tuned to reproduce the performance of the ATLAS detector [50, 51]. The working point for jet $b$-tagging has an efficiency of $77 \%$, with a rejection factor $\sim 5$ and $\sim 110$ respectively on $c$ and light jets. 


\subsection{Statistical procedures}

The LHC sensitivity to the three proposed signatures is estimated for integrated luminosities of $140 \mathrm{fb}^{-1}, 300 \mathrm{fb}^{-1}$ and $3000 \mathrm{fb}^{-1}$, corresponding to the available statistics of LHC Run 2 and the projected statistics for LHC Run 3 and the high-luminosity LHC run respectively. We assume the same detector performances for the high-luminosity LHC as for the previous data-taking runs.

The sensitivity is calculated using a test statistics based on a profiled likelihood ratio, and we make use of the CLs method [52] to obtain 95\% confidence level (CL) exclusion limits. The statistical analysis is performed with the RooSTAT toolkit [53] and we assume systematic uncertainties of $15 \%$ and $5 \%$ on the SM backgrounds and on the signal respectively.

\subsection{Analysis strategy}

All of the addressed signatures, monotop, $t j$ and $t b$, have a single top quark in the final state, and we consider its semileptonic decay. Therefore, the common basic selection for all three signatures is the requirement of one and only one isolated lepton ( $e$ or $\mu)$ with $p_{T}>30 \mathrm{GeV}$ within $|\eta|<2.5$. Considering the monotop signature, the signal has a strong imbalance in favour of a positive lepton, therefore only leptons with positive charge are considered. For all signatures, we further require the presence of at least one $b$-tagged jet and of $E_{\mathrm{T}}^{\mathrm{miss}}$ from neutrinos and dark matter escaping the detector. The requirements on additional jet activity depend on the signature. For monotop and $t j$, one and only one $b$-tagged jet is required, while for $t b$ two $b$-tagged jets are required. Additional jets with $p_{T}>50 \mathrm{GeV}$ are vetoed for monotop and $t b$, whereas an additional high $p_{T}$ jet in the event is expected for $t j$, from the decay of $\phi$ into a light jet.

For events featuring a semileptonic decay of the top, the invariant mass of the lepton and the $b$-tagged jet $m_{b \ell}$ has a sharp end-point around $160 \mathrm{GeV}$. For the $t b$ analysis, where two $b$-tagged jets are required, the minimum of the two $b$-lepton invariant mass combinations has an equivalent property. An upper limit at $160 \mathrm{GeV}$ on this variable ensures a significant reduction of non-top backgrounds.

The above requirements will select also an overwhelming number of background events from standard model processes featuring the production of a $W$ boson decaying into leptons, dominated by $t \bar{t}$ and $W+$ jets production. The main handle against these backgrounds is the fact that they have $E_{\mathrm{T}}^{\text {miss }}$ only from the neutrino from $W$ decay, whereas the signal has a large additional $E_{\mathrm{T}}^{\text {miss }}$ from the invisible $\chi$ particles. A useful variable to exploit this feature is built out of the transverse momentum of the lepton and $E_{\mathrm{T}}^{\mathrm{miss}}$ as:

$$
m_{\mathrm{T}}^{\text {lep }} \equiv \sqrt{2\left|\vec{p}_{\mathrm{T}}^{\ell}\right| E_{\mathrm{T}}^{\text {miss }}\left(1-\cos \Delta \phi\left(\vec{p}_{\mathrm{T}}^{\ell}, \vec{p}_{\mathrm{T}}^{\text {miss }}\right)\right)},
$$

where $\vec{p}_{\mathrm{T}}^{\ell}$ is the transverse component of the momentum of the lepton, and $\vec{p}_{\mathrm{T}}^{\text {miss }}$ is defined in the previous section. For events where the lepton and all of the $E_{\mathrm{T}}^{\text {miss }}$ are produced in the decay of a single $W$, this variable is bounded from above by the $W$ mass. For the $t j$ and $t b$ signatures a lower limit of $160 \mathrm{GeV}$ on this variable reduces then the single $W$ backgrounds to a manageable level. For monotop, which is a simpler final state with less kinematic handles to reduce the backgrounds, a stronger limit of $250 \mathrm{GeV}$ on $m_{\mathrm{T}}^{\text {lep }}$ is applied. 
The dominant background after an appropriate $m_{\mathrm{T}}^{\text {lep }}$ requirement is typically composed of $t \bar{t}$ events where both tops decay semileptonically and only one lepton is detected. The by now standard approach to reject this background is the asymmetric $m_{\mathrm{T} 2}$ variable (denoted $\left.a m_{\mathrm{T} 2}\right)[54,55]$ that consists in a variant of the $m_{\mathrm{T} 2}$ observable. The $a m_{\mathrm{T} 2}$ variable is built from two legs (corresponding to the two decay chains) containing both a visible part and an invisible part, and it requires two test masses corresponding to the invisible mass attached with each leg. For the $t \bar{t}$ 2-lepton background with one lepton lost, the visible part of the first leg is the vector sum of the momenta of the $b$-tagged jet and of the lepton, with a test mass set to zero. The visible part of the second leg is built choosing among the additional jets in the event the one with the highest $b$-tagging weight, and the test mass is set to $80 \mathrm{GeV}$. Of course a selection on this variable cannot be applied for monotop, where only the $b$-jet from the decay of the top is allowed in the event.

After removing the background events within the $W$ kinematic bounds, there is still a significant background from events where a large $E_{\mathrm{T}}^{\mathrm{miss}}$ is produced by the mismeasurement of a jet. These events have the $E_{\mathrm{T}}^{\text {miss }}$ aligned with the momentum of a jet. The $\Delta \phi_{\text {min }}$ variable is built as the minimal angular difference in the transverse plane between $E_{\mathrm{T}}^{\text {miss }}$ and any reconstructed jet in the event. This variable has an enhancement near zero for the background, and a lower limit on it increases the signal over background ratio.

The angular differences in the plane transverse to the beam between pairs of observed objects can provide discrimination power between signal and background. The variables used in the analysis are $\Delta \phi_{b \ell}$ and $\Delta \phi_{m \ell}$, the angular difference of the lepton with the $b$-jet and $E_{\mathrm{T}}^{\text {miss }}$, respectively. For the $t b$ analysis the former variable is built with the $b$-jet most likely to be from the top decay. Harder cuts on the angular variables are applied for the monotop signature with respect to $t j$ and $t b$, again to compensate for the less constrained kinematics of the signature.

Finally, the $t j$ analysis addresses the signal topology featuring one leg where a heavy mediator decays into a hard jet and dark matter. In this case the distribution in the transverse mass built from the transverse momentum of the hard jet and the one of the $\chi$ particles has an end-point at $\left(m_{\phi}^{2}-m_{\chi}^{2}\right)^{1 / 2}$. This feature can be exploited by defining a dedicated variation on $a m_{\mathrm{T} 2}$ which we call the $m_{\mathrm{T} 2} b_{l j}$ variable. The visible part of the first leg is built from the sum of the momenta of the $b$-tagged jet and of the lepton, and the test mass is set to zero. The visible part of the second leg uses the hardest non- $b$-tagged jet, and again a zero test mass.

The selection criteria for the signal regions for the three analyses, based on the variables defined above, are given in table 2. For all three signatures, the final sensitivity is calculated for each signal point considered by varying the cut value on the final discriminant variable, which is $E_{\mathrm{T}}^{\text {miss }}$ for monotop, $m_{\mathrm{T} 2} b l j$ for $t j$ and $a m_{\mathrm{T} 2}$ for $t b$.

The distributions of the final discriminant variable after all other cuts have been applied are shown in figure 12 for the backgrounds and for benchmark signal samples for the $t j$ and monotop analyses.

For $m_{\phi}=1 \mathrm{TeV}$ and a cut of $400 \mathrm{GeV}$ on the final discriminant, the signal efficiency is between 0.7 and $1 \%$ for monotop, with a background of $\sim 60$ events for $300 \mathrm{fb}^{-1}$. For $t j$, the efficiency is between 3 and $5 \%$ and the background of $\sim 50$ events. For $t b$, the efficiency is between $1 \%$ and $1.5 \%$ for a background of $\sim 6$ events. 


\begin{tabular}{|lccc|}
\hline Variable [Unit] & monotop & $t j$ & $t b$ \\
\hline$N_{\ell}$ & $=1$ & $=1$ & $=1$ \\
$p_{T}(\ell)[\mathrm{GeV}]$ & $>30$ & $>30$ & $>30$ \\
$\operatorname{charge}(\ell)$ & $>0$ & any & any \\
$N_{b \text {-jet }}$ & $=1$ & $=1$ & $=2$ \\
$p_{T}\left(b_{1}\right)[\mathrm{GeV}]$ & $>30$ & $>30$ & $>110$ \\
$p_{T}\left(b_{2}\right)[\mathrm{GeV}]$ & - & - & $>110$ \\
$p_{T}(j)[\mathrm{GeV}]$ & $<50$ & $>100$ & $<50$ \\
$m_{b \ell}[\mathrm{GeV}]$ & $<160$ & $<160$ & $<160$ \\
$m_{\mathrm{T}}^{\text {lep }}[\mathrm{GeV}]$ & $>250$ & $>160$ & $>160$ \\
$\Delta \phi_{\mathrm{min}}[\mathrm{rad}]$ & $>2$ & $>0.6$ & $>0.7$ \\
$\Delta \phi_{b \ell}[\mathrm{rad}]$ & $<1.5$ & incl & $<1.5$ \\
$\Delta \phi_{m \ell}[\mathrm{rad}]$ & $>2.5$ & incl. & incl. \\
$E_{\mathrm{T}}^{\mathrm{miss}}[\mathrm{GeV}]$ & $>400-600$ & $>90$ & $>250$ \\
$a m_{\mathrm{T} 2}[\mathrm{GeV}]$ & - & $>250$ & $>300-500$ \\
$m_{\mathrm{T} 2 b l j}[\mathrm{GeV}]$ & - & $>300-500$ & - \\
\hline
\end{tabular}

Table 2. Summary of the selection criteria for the three proposed single-top analyses. The cuts on $E_{\mathrm{T}}^{\mathrm{miss}}$ and $m_{\mathrm{T} 2_{b l j}}$ respectively for the monotop and $t j$ analyses are optimised separately for each value of $m_{\phi}$ within the range given in the table. The variable $p_{T}(j)$ is the transverse momentum of the hardest jet not tagged as a $b$-jet.
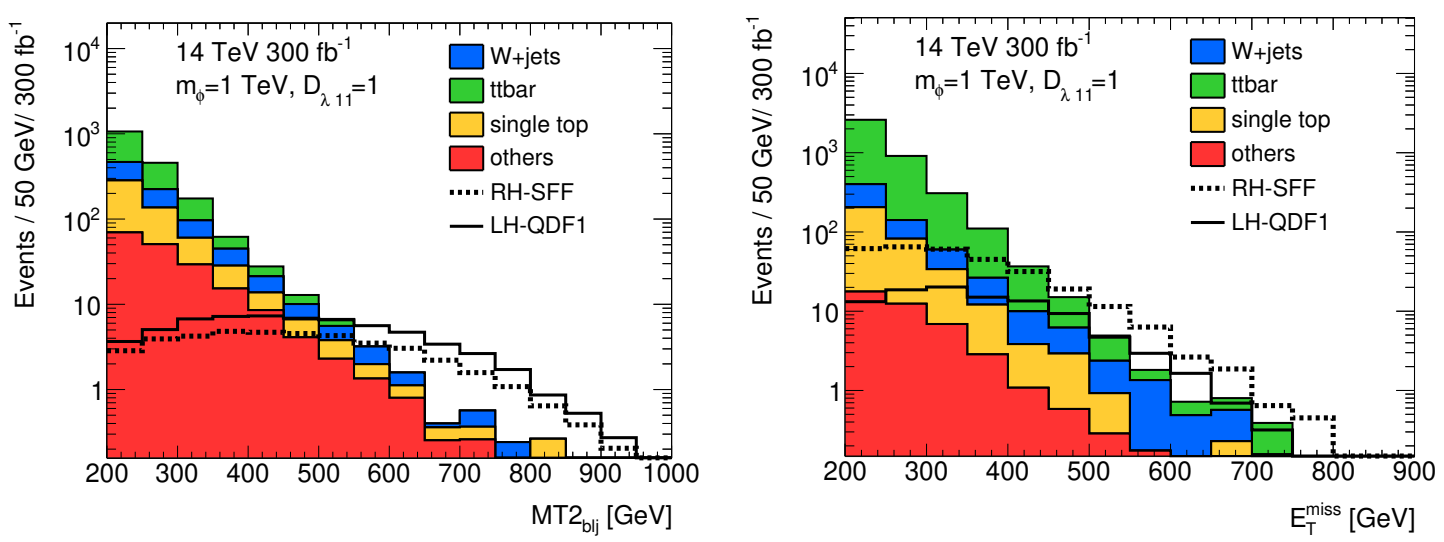

Figure 12. Distribution of the $m_{\mathrm{T} 2}$ (left) and $E_{\mathrm{T}}^{\text {miss }}$ (right) variables for the $t j$ and monotop analyses respectively for two signal points and for the SM backgrounds. The distributions are shown after all of the selection cuts are applied except the one on the plotted variable. The normalisation corresponds to an integrated luminosity of $300 \mathrm{fb}^{-1}$.

The efficiency of the analysis strategies outlined above is only mildly dependent on $m_{\phi}$, but it displays a characteristic threshold dependence on the mass difference $\Delta m \equiv m_{\phi}-m_{\chi}$. For fixed $m_{\phi}$ and $\Delta m>700-800 \mathrm{GeV}$, the efficiency is approximately independent from 
$\Delta m$, whereas it quickly decreases when $\Delta m$ decreases below the threshold value. For low $\Delta m$ the visible decay products of $\phi$ become soft, failing the kinematic requirements which are necessary to suppress to an adequate level the SM backgrounds. Therefore the results shown below will apply to different choices of $m_{\chi}$ than the ones corresponding to our benchmarks for values of $m_{\phi}$ such that $\Delta m$ is above threshold.

Based on these results and on the dependence of the production cross-section of the signatures on the model parameters, we can explore in the next section the relative importance of the different signatures over the parameter space of the model for each of the four benchmarks.

\section{$6 \quad$ Results}

For each of the proposed benchmarks we compare the area excluded by the CMS analysis in the $\left(m_{\phi}, D_{\lambda, 11}\right)$ plane based on $137 \mathrm{fb}^{-1}$ of $13 \mathrm{TeV}$ LHC data to the area covered on the same plane by each of the single top analyses proposed in this paper for 137 and $300 \mathrm{fb}^{-1}$ of $14 \mathrm{TeV}$ LHC data. The purpose is to verify whether the new analyses would provide a gain in coverage of the parameter space of the model with respect to the existing flavour-conserving SUSY searches, and to gauge the expected improvement with the doubled luminosity expected at the end of the LHC Run 3. We note that the sensitivities for $137 \mathrm{fb}^{-1}$ are shown for a $14 \mathrm{TeV} \mathrm{LHC}$, whereas the CMS results are for $13 \mathrm{TeV}$, therefore the comparison is not fully consistent. It is anyway useful to give an idea, with a comparable amount of data, of the relative power of the different analyses in different regions of the parameter space.

The results are shown for the four benchmarks in figure 13, where the presently excluded region is shown as a shaded area, and the coverage of the single top analyses is shown as full (dashed) lines for $137(300) \mathrm{fb}^{-1}$.

In RH-SFF, the coupling to the third generation dominates over the one to the first generation, and a dedicated monotop analysis would increase the reach in mediator mass for $D_{\lambda, 11}>0.4$, with the $t j$ analysis covering region similar to the one already excluded by CMS for $D_{\lambda, 11}>0.6$. Already with $137 \mathrm{fb}^{-1}$ the monotop analysis would probe the parameter space predicted by thermal freeze-out. For low values of $D_{\lambda, 11}$, none of the single top analyses would improve on the CMS limits from the stop analysis.

For RH-QDF, where $D_{\lambda, 11}$ and $D_{\lambda, 33}$ have similar values, the flavour conserving analyses display a minimum in the mass coverage for $D_{\lambda, 11} \sim 0.6$, corresponding to the situation where the $t t$ and $j j$ signatures have both a $25 \% \mathrm{BR}$. The $t j$ signature for low $D_{\lambda, 11}$ is dominated by the doubly resonant production of the mediator, with one mediator decaying into a light quark and the other mediator decaying into top, with a $50 \%$ BR approximately constant for $D_{\lambda, 11}>0.2$, as discussed in section 3.1. As a result, the $t j$ analysis increases significantly the coverage with respect to the flavour-conserving searches, with an approximately flat mass reach of $\sim 900 \mathrm{GeV}$ in the $D_{\lambda, 11}$ interval from $\sim 0.1$ to $\sim 0.6$. For higher values of $D_{\lambda, 11}$, the $t$-channel production becomes dominant and the mass reach approaches the one of the jet-jet CMS analysis. The monotop analysis gives the best reach for 

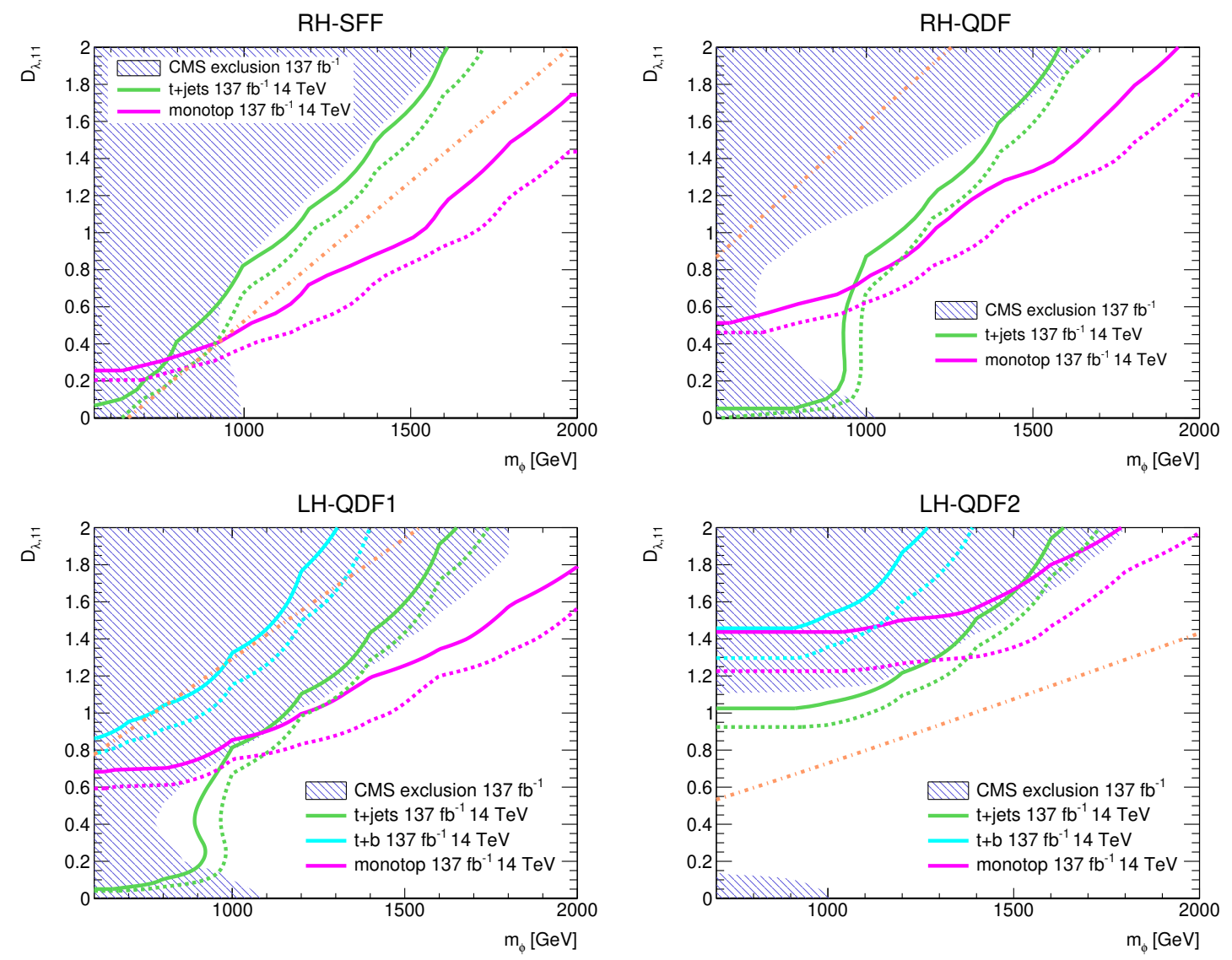

Figure 13. Full lines: excluded areas in the $\left(m_{\phi}, D_{\lambda, 11}\right)$ plane for the $t j, t b$ and monotop analyses proposed in this paper for an integrated luminosity of $137 \mathrm{fb}^{-1}$ at a $14 \mathrm{TeV}$ LHC. The excluded areas are on the left of the curves. The dashed lines show the corresponding results for $300 \mathrm{fb}^{-1}$. The shaded area corresponds to the region excluded by the CMS analyses. The orange dash-dotted lines indicate for which set of parameters the correct relic abundance is obtained.

$D_{\lambda, 11}>0.7$, improving by several hundreds of $\mathrm{GeV}$ the mediator mass reach with respect to the flavour-conserving analyses.

The benchmark LH-QDF1 is very similar to RH-QDF, with the main difference that the mediator in this case couples both with up and down quark flavours, thus altering the relative branching ratios and opening up channels with $b$-jets in the final state. The pattern of mass reach of the different channels indeed approximately matches the one for RH-QDF1, with the $t j$ channel increasing the mass reach in the region where the flavourconserving exclusion has a minimum, and with the dominance of monotop at high $D_{\lambda, 11}$. The additional $t b$ channel has sensitivity only in regions already excluded by the CMS searches.

The phenomenology of the LH-QDF2 benchmark, as discussed above, is dominated by the high value of $m_{\chi}=450 \mathrm{GeV}$. The single top channels cover marginally the range of $D_{\lambda, 11}$ couplings for which the existing analyses have no sensitivity. The $t j$ analysis excludes the uncovered region with $D_{\lambda, 11}$ between $\sim 1.0$ and $\sim 1.1$ for the lowest allowed mediator masses. The monotop and the $t b$ analyses only cover the area already excluded by CMS. 

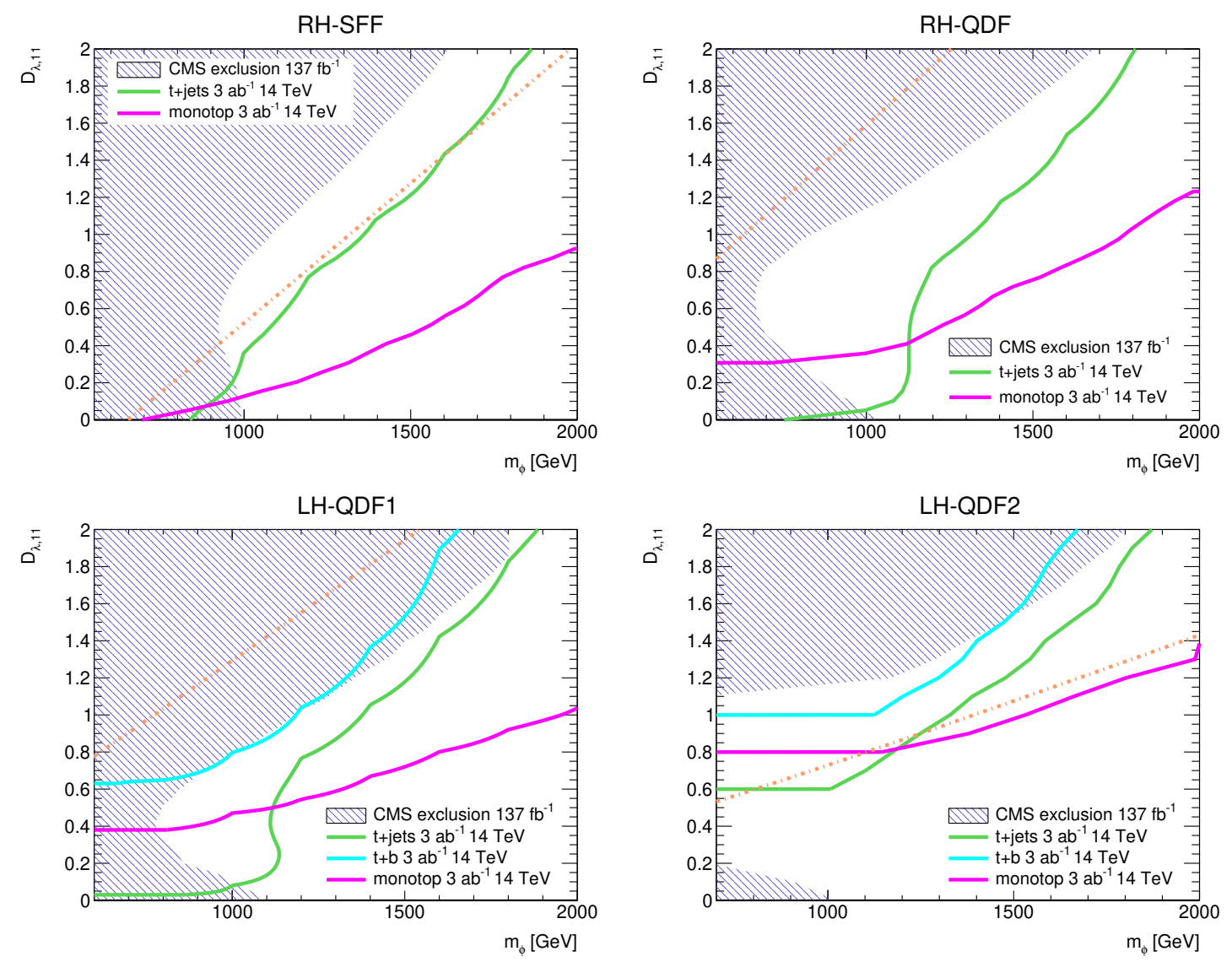

Figure 14. Excluded areas in the $\left(m_{\phi}, D_{\lambda, 11}\right)$ plane for the $t j, t b$ and monotop analyses proposed in this paper for an integrated luminosity of $3 \mathrm{ab}^{-1}$ at a $14 \mathrm{TeV}$ LHC. The excluded areas are on the left of the curves. The shaded area corresponds to the region excluded by the CMS analyses. The orange dash-dotted lines indicate for which set of parameters the correct relic abundance is obtained.

The curves for $300 \mathrm{fb}^{-1}$ follow closely the ones for $137 \mathrm{fb}^{-1}$, with a gain between 0.1 and 0.3 units of $D_{\lambda, 11}$ depending on the signature and on the mediator mass. The monotop reach in LH-QDF2 is statistics limited, and the doubling of the statistics yields a somewhat larger improvement than for the other channels/benchmarks.

In figure 14 the statistics of the HL-LHC $\left(3000 \mathrm{fb}^{-1}\right)$ are shown, based on the statistical procedure defined above. For all of the benchmarks the monotop analysis will cover mediator masses of $2 \mathrm{TeV}$ for $D_{\lambda, 11}$ varying between 0.8 and 1.2 dependent on the benchmark. For lower values of the couplings, the impact of the single top analyses depends on the specific benchmark.

Another common feature is that for values of $D_{\lambda, 11} \leq 0.1$ even at the HL-LHC the single top analyses will not be able to improve on the CMS exclusion limit. In the low $D_{\lambda, 11}$ region the dominant sensitivity will always be provided by the SUSY stop searches.

For RH-SFF, the monotop search will always provide the best mass coverage among the proposed analyses, but it will compete with the HL-LHC stop searches for low $D_{\lambda, 11}$. 
In the case of RH-QDF and LH-QDF1, the excluded mediator mass in the region between $D_{\lambda, 11}=0.1$ and $D_{\lambda, 11}=0.8$, which are difficult for flavour-conserving analyses, will be $\sim 1150 \mathrm{GeV}$, but monotop will provide better coverage starting from $D_{\lambda, 11} \geq 0.5$.

The uncovered region in $D_{\lambda, 11}$ for LH-QDF2 will be reduced to the interval between 0.1 and 0.6 by the HL-LHC searches. The monotop signature will provide sensitivity up to $m_{\phi} \sim 1600 \mathrm{GeV}$ for $D_{\lambda, 11}=1$ and will probe the thermal freeze-out scenario for mediator masses above $1100 \mathrm{GeV}$. Between 800 and $1200 \mathrm{GeV}$ the thermal relic assumption will be tested by the $t j$ analysis.

\section{Conclusion and outlook}

Abundant production of final states with two quarks of different flavours accompanied by $E_{\mathrm{T}}^{\text {miss }}$ from dark matter particles is a well defined prediction of flavoured DM models within the DMFV framework. A particularly interesting case is when one of the two quarks is a top quark, which, with its distinctive decay signature, provides an excellent experimental handle for searching for this model at colliders.

In the present study, we addressed three signatures with a single top in the final state, accompanied by $E_{\mathrm{T}}^{\mathrm{miss}}$ and by no additional jet or a light jet or a $b$-jet. For each of these signatures, we fully developed a search strategy at a $14 \mathrm{TeV}$ LHC, taking into account all relevant standard model backgrounds. The potential of the proposed new searches was tested on four phenomenologically viable benchmark models with only two free parameters, where all the remaining parameters of the model are fixed by low-energy or astrophysical constraints. We compared the reach of the proposed signatures with the recast of existing flavour-conserving SUSY limits obtained at the LHC with $13 \mathrm{TeV}$ center-of-mass energy and an integrated luminosity of $137 \mathrm{fb}^{-1}$.

It turns out that for all of the considered benchmarks the final states with a single top do increase the reach of the existing analyses in the considered parameter space, and there are large regions in parameter space where the signal from several different analyses would be detectable, providing a handle on model discrimination. A projection of the reach of the proposed analyses to the full LHC luminosity and to the luminosity of the HL-LHC shows that for all of the benchmark models mediator masses of $1.6 \mathrm{TeV}$ or above can be probed for a DM coupling parameter $D_{\lambda, 11}=1$, with the reach decreasing for lower values of $D_{\lambda, 11}$, where the final state with two top quarks and $E_{\mathrm{T}}^{\text {miss }}$ will eventually be the most sensitive channel.

A monotop analysis optimised for these models along with searches for single top quarks accompanied by jets would hence provide the experimental collaborations with a window to dark matter models incorporating flavour violation, which have received only passing attention in the early LHC analyses.

\section{Acknowledgments}

The research of MB is supported by the DFG Collaborative Research Center TRR 257 "Particle Physics Phenomenology after the Higgs Discovery". 
Open Access. This article is distributed under the terms of the Creative Commons Attribution License (CC-BY 4.0), which permits any use, distribution and reproduction in any medium, provided the original author(s) and source are credited.

\section{References}

[1] G. Arcadi et al., The waning of the WIMP? A review of models, searches, and constraints, Eur. Phys. J. C 78 (2018) 203 [arXiv:1703.07364] [INSPIRE].

[2] L. Roszkowski, E.M. Sessolo and S. Trojanowski, WIMP dark matter candidates and searches - current status and future prospects, Rept. Prog. Phys. 81 (2018) 066201 [arXiv: 1707.06277] [INSPIRE].

[3] J. Kile and A. Soni, Flavored Dark Matter in Direct Detection Experiments and at LHC, Phys. Rev. D 84 (2011) 035016 [arXiv:1104.5239] [InSPIRE].

[4] J.F. Kamenik and J. Zupan, Discovering Dark Matter Through Flavor Violation at the LHC, Phys. Rev. D 84 (2011) 111502 [arXiv:1107.0623] [INSPIRE].

[5] B. Batell, J. Pradler and M. Spannowsky, Dark Matter from Minimal Flavor Violation, JHEP 08 (2011) 038 [arXiv:1105.1781] [INSPIRE].

[6] P. Agrawal, S. Blanchet, Z. Chacko and C. Kilic, Flavored Dark Matter, and Its Implications for Direct Detection and Colliders, Phys. Rev. D 86 (2012) 055002 [arXiv:1109.3516] [INSPIRE].

[7] B. Batell, T. Lin and L.-T. Wang, Flavored Dark Matter and R-Parity Violation, JHEP 01 (2014) 075 [arXiv: 1309.4462] [InSPIRE].

[8] J. Kile, Flavored Dark Matter: A Review, Mod. Phys. Lett. A 28 (2013) 1330031 [arXiv: 1308.0584] [INSPIRE].

[9] L. Lopez-Honorez and L. Merlo, Dark matter within the minimal flavour violation ansatz, Phys. Lett. B $\mathbf{7 2 2}$ (2013) 135 [arXiv:1303.1087] [INSPIRE].

[10] A. Kumar and S. Tulin, Top-flavored dark matter and the forward-backward asymmetry, Phys. Rev. D 87 (2013) 095006 [arXiv:1303.0332] [INSPIRE].

[11] Y. Zhang, Top Quark Mediated Dark Matter, Phys. Lett. B 720 (2013) 137 [arXiv: 1212.2730] [INSPIRE].

[12] P. Agrawal, M. Blanke and K. Gemmler, Flavored dark matter beyond Minimal Flavor Violation, JHEP 10 (2014) 072 [arXiv: 1405.6709] [INSPIRE].

[13] M. Blanke and S. Kast, Top-Flavoured Dark Matter in Dark Minimal Flavour Violation, JHEP 05 (2017) 162 [arXiv: 1702.08457] [INSPIRE].

[14] M. Blanke, S. Das and S. Kast, Flavoured Dark Matter Moving Left, JHEP 02 (2018) 105 [arXiv:1711.10493] [INSPIRE].

[15] M.-C. Chen, J. Huang and V. Takhistov, Beyond Minimal Lepton Flavored Dark Matter, JHEP 02 (2016) 060 [arXiv:1510.04694] [INSPIRE].

[16] A.J. Buras, P. Gambino, M. Gorbahn, S. Jäger and L. Silvestrini, Universal unitarity triangle and physics beyond the standard model, Phys. Lett. B 500 (2001) 161 [hep-ph/0007085] [INSPIRE].

[17] G. D'Ambrosio, G.F. Giudice, G. Isidori and A. Strumia, Minimal flavor violation: An Effective field theory approach, Nucl. Phys. B 645 (2002) 155 [hep-ph/0207036] [INSPIRE]. 
[18] A.J. Buras, Minimal flavor violation, Acta Phys. Polon. B 34 (2003) 5615 [hep-ph/0310208] [INSPIRE].

[19] R.S. Chivukula and H. Georgi, Composite Technicolor Standard Model, Phys. Lett. B 188 (1987) 99 [INSPIRE].

[20] L.J. Hall and L. Randall, Weak scale effective supersymmetry, Phys. Rev. Lett. 65 (1990) 2939 [INSPIRE].

[21] T. Jubb, M. Kirk and A. Lenz, Charming Dark Matter, JHEP 12 (2017) 010 [arXiv: 1709.01930] [INSPIRE].

[22] C. Kilic, M.D. Klimek and J.-H. Yu, Signatures of Top Flavored Dark Matter, Phys. Rev. D 91 (2015) 054036 [arXiv: 1501.02202] [INSPIRE].

[23] J. Andrea, B. Fuks and F. Maltoni, Monotops at the LHC, Phys. Rev. D 84 (2011) 074025 [arXiv:1106.6199] [INSPIRE].

[24] E. Alvarez, E. Coluccio Leskow, J. Drobnak and J.F. Kamenik, Leptonic Monotops at LHC, Phys. Rev. D 89 (2014) 014016 [arXiv:1310.7600] [INSPIRE].

[25] ATLAS collaboration, Search for large missing transverse momentum in association with one top-quark in proton-proton collisions at $\sqrt{s}=13 \mathrm{TeV}$ with the ATLAS detector, JHEP 05 (2019) 041 [arXiv: 1812.09743] [INSPIRE].

[26] CMS collaboration, Search for dark matter produced in association with a single top quark or a top quark pair in proton-proton collisions at $\sqrt{s}=13 \mathrm{TeV}$, JHEP 03 (2019) 141 [arXiv: 1901.01553] [INSPIRE].

[27] T. Hurth and W. Porod, Flavour violating squark and gluino decays, JHEP 08 (2009) 087 [arXiv: 0904.4574] [INSPIRE].

[28] M. Blanke, G.F. Giudice, P. Paradisi, G. Perez and J. Zupan, Flavoured Naturalness, JHEP 06 (2013) 022 [arXiv: 1302.7232] [INSPIRE].

[29] A. Chakraborty et al., Flavour-violating decays of mixed top-charm squarks at the LHC, Eur. Phys. J. C 78 (2018) 844 [arXiv: 1808.07488] [InSPIRE].

[30] ATLAS collaboration, Search for supersymmetry in events with b-tagged jets and missing transverse momentum in pp collisions at $\sqrt{s}=13 \mathrm{TeV}$ with the ATLAS detector, JHEP 11 (2017) 195 [arXiv: 1708. 09266] [INSPIRE].

[31] CMS collaboration, Search for supersymmetry in proton-proton collisions at $13 \mathrm{TeV}$ in final states with jets and missing transverse momentum, JHEP 10 (2019) 244

[arXiv: 1908. 04722] [INSPIRE].

[32] CMS collaboration, Search for direct top squark pair production in events with one lepton, jets, and missing transverse momentum at $13 \mathrm{TeV}$ with the CMS experiment, JHEP 05 (2020) 032 [arXiv: 1912.08887] [INSPIRE].

[33] CMS collaboration, Searches for physics beyond the standard model with the $M_{\mathrm{T} 2}$ variable in hadronic final states with and without disappearing tracks in proton-proton collisions at $\sqrt{s}=13$ TeV, Eur. Phys. J. C 80 (2020) 3 [arXiv:1909.03460] [INSPIRE].

[34] ATLAS collaboration, Search for a scalar partner of the top quark in the all-hadronic t $\bar{t}$ plus missing transverse momentum final state at $\sqrt{s}=13 \mathrm{TeV}$ with the ATLAS detector, Eur. Phys. J. C 80 (2020) 737 [arXiv:2004.14060] [InSPIRE]. 
[35] C. Degrande, C. Duhr, B. Fuks, D. Grellscheid, O. Mattelaer and T. Reiter, UFO - The Universal FeynRules Output, Comput. Phys. Commun. 183 (2012) 1201 [arXiv:1108.2040] [INSPIRE].

[36] J. Alwall et al., The automated computation of tree-level and next-to-leading order differential cross sections, and their matching to parton shower simulations, JHEP 07 (2014) 079 [arXiv: 1405.0301] [INSPIRE].

[37] NNPDF collaboration, Parton distributions for the LHC Run II, JHEP 04 (2015) 040 [arXiv: 1410.8849] [INSPIRE].

[38] T. Sjöstrand et al., An introduction to PYTHIA 8.2, Comput. Phys. Commun. 191 (2015) 159 [arXiv: 1410.3012] [INSPIRE].

[39] J.M. Campbell, R.K. Ellis, P. Nason and E. Re, Top-Pair Production and Decay at NLO Matched with Parton Showers, JHEP 04 (2015) 114 [arXiv: 1412.1828] [INSPIRE].

[40] E. Re, Single-top Wt-channel production matched with parton showers using the POWHEG method, Eur. Phys. J. C 71 (2011) 1547 [arXiv:1009.2450] [InSPIRE].

[41] T. Melia, P. Nason, R. Rontsch and G. Zanderighi, $W^{+} W^{-}, W Z$ and $Z Z$ production in the POWHEG BOX, JHEP 11 (2011) 078 [arXiv:1107.5051] [INSPIRE].

[42] P. Nason and G. Zanderighi, $W^{+} W^{-}, W Z$ and $Z Z$ production in the POWHEG-BOX-V2, Eur. Phys. J. C $\mathbf{7 4}$ (2014) 2702 [arXiv:1311.1365] [InSPIRE].

[43] S. Alioli, P. Nason, C. Oleari and E. Re, A general framework for implementing NLO calculations in shower Monte Carlo programs: the POWHEG BOX, JHEP 06 (2010) 043 [arXiv: 1002.2581] [INSPIRE].

[44] M. Czakon and A. Mitov, Top++: A Program for the Calculation of the Top-Pair Cross-Section at Hadron Colliders, Comput. Phys. Commun. 185 (2014) 2930 [arXiv:1112.5675] [INSPIRE].

[45] M. Czakon, P. Fiedler and A. Mitov, Total Top-Quark Pair-Production Cross Section at Hadron Colliders Through $O\left(\alpha_{S}^{4}\right)$, Phys. Rev. Lett. 110 (2013) 252004 [arXiv:1303.6254] [INSPIRE].

[46] C. Anastasiou, L.J. Dixon, K. Melnikov and F. Petriello, High precision QCD at hadron colliders: Electroweak gauge boson rapidity distributions at NNLO, Phys. Rev. D 69 (2004) 094008 [hep-ph/0312266] [INSPIRE].

[47] R. Gavin, Y. Li, F. Petriello and S. Quackenbush, W Physics at the LHC with FEWZ 2.1, Comput. Phys. Commun. 184 (2013) 208 [arXiv:1201.5896] [INSPIRE].

[48] M. Cacciari, G.P. Salam and G. Soyez, The anti- $k_{t}$ jet clustering algorithm, JHEP 04 (2008) 063 [arXiv: 0802.1189] [INSPIRE].

[49] M. Cacciari, G.P. Salam and G. Soyez, FastJet User Manual, Eur. Phys. J. C 72 (2012) 1896 [arXiv: 1111.6097] [inSPIRE].

[50] ATLAS collaboration, The ATLAS Experiment at the CERN Large Hadron Collider, 2008 JINST 3 S08003 [INSPIRE].

[51] ATLAS collaboration, Expected Performance of the ATLAS Experiment - Detector, Trigger and Physics, arXiv:0901.0512 [INSPIRE].

[52] A.L. Read, Presentation of search results: The CL technique, J. Phys. G 28 (2002) 2693 [INSPIRE]. 
[53] L. Moneta et al., The RooStats Project, PoS ACAT2010 (2010) 057 [arXiv:1009.1003] [INSPIRE].

[54] P. Konar, K. Kong, K.T. Matchev and M. Park, Dark Matter Particle Spectroscopy at the LHC: Generalizing $M_{T 2}$ to Asymmetric Event Topologies, JHEP 04 (2010) 086 [arXiv:0911.4126] [INSPIRE].

[55] C.G. Lester and B. Nachman, Bisection-based asymmetric $M_{T 2}$ computation: a higher precision calculator than existing symmetric methods, JHEP 03 (2015) 100 [arXiv: 1411.4312] [INSPIRE]. 